\title{
Coset approach to the partial breaking of global supersymmetry
}

\author{
S. Bellucci ${ }^{a}$, S. Krivonos ${ }^{b}$, A. Sutulin ${ }^{a, b}$ \\ ${ }^{a}$ INFN-Laboratori Nazionali di Frascati, Via E. Fermi 40, 00044 Frascati, Italy \\ ${ }^{b}$ Bogoliubov Laboratory of Theoretical Physics, JINR, 141980 Dubna, Russia
}

\begin{abstract}
We propose a method to construct the on-shell component actions for the theories with $1 / 2$ partial breaking of global supersymmetry within the nonlinear realization (coset) approach. In contrast with the standard superfield approach in which unbroken supersymmetry plays the leading role, we have shifted the attention to the spontaneously broken supersymmetry. It turns out that in the theories in which one half of supersymmetries is spontaneously broken, all physical fermions are just the fermions of the nonlinear realization. Moreover, the transformation properties of these fermions with respect to the broken supersymmetry are the same as in the famous Volkov-Akulov model. Just this fact completely fixed all possible appearances of the fermions in the component action: they can enter the action through the determinant of the vielbein (to compensate the transformation of the volume form) and the covariant derivatives, only. It is very important that in our parametrization of the coset the rest of physical components, i.e. all bosonic components, transform as "matter fields" with respect to the broken supersymmetry. Clearly, in such a situation the component action acquires the form of the Volkov-Akulov action for these "matter fields". The complete form of the action can be further fixed by two additional requirements: a) to reproduce the bosonic limit, which is explicitly known in many interesting cases, and b) to have a proper linearized form, which has to be invariant with respect to the linearized unbroken supersymmetry. In some cases the additional Wess-Zumino terms (which disappear in the bosonic limit) have to be added to the action. We supply the general consideration by the detailed examples of the actions for the superparticle in $D=3$ realizing $N=4 \cdot 2^{k} \rightarrow N=2 \cdot 2^{k}$ pattern of supersymmetry breaking, the superparticle in $D=5$ with the $N=16$ supersymmetry broken down to $N=8$ one, the on-shell component action for $N=1, D=5$ supermembrane and its dual cousins and the component action of $N=1$ supermembrane in $D=4$. In these cases we provide the exact proof of the invariance of the constructed component actions with respect to both broken and unbroken supersymmetries.
\end{abstract}




\section{Introduction}

The characteristic feature of the theories with a partial breaking of global supersymmetries is the appearance of the Goldstone fermionic fields, associated with the broken supertranslations, as the components of Goldstone supermultiplets of unbroken supersymmetry. The natural description of such theories is achieved within the coset approach [1, 2, 3]. The usefulness of the coset approach in the applications to the theories with partial breaking of the supersymmetry have been demonstrated by many authors [4-20]. The presence of the unbroken supersymmetry makes quite reasonable the idea to choose the corresponding superfields as the basic ones, and many interesting superspace actions describing different patterns of supersymmetry breaking have been constructed in such a way [4, 7, 8, 9]. However, the standard methods of coset approach fail to construct the superfield action, because the superspace Lagrangian is weakly invariant with respect to supersymmetry - it is shifted by the full space-time or spinor derivatives under broken/unbroken supersymmetry transformations. Another rather technical difficulty is the explicit construction of the component action from the superspace one, which is written in terms of the superfields subjected to highly nonlinear constraints. Finally, in some cases the covariantization of the irreducibility constraints with respect to the broken supersymmetry is not evident, if at all possible. For example, it has been demonstrated in [4] that such constraints for the vector supermultiplet can be covariantized only together with the equations of motion.

It turned out that one can gain more information about component off-shell actions if an attention is shifted to the broken supersymmetry. It was demonstrated in [17, [18, [19] that with a suitable choice of the parametrization of the coset, the $\theta$-coordinates of unbroken supersymmetry and the physical bosonic components do not transform under broken supersymmetry. Moreover, the physical fermions transform as the Goldstino of the Volkov-Akulov model 3 with respect to broken supersymmetry. Therefore, the physical fermions can enter the component on-shell action only i) through the determinant of the vielbein (to compensate the variation of the volume $d^{d} x$ ), ii) through the covariant space-time derivatives, or iii) through the Wess-Zumino term, if it exists. The first two ingredients can be easily constructed within the coset method, while the Wess-Zumino term can be also constructed from Cartan forms following the recipe of ref. [21. As a result, we will have the Ansatz for the action with several constant parameters, which have to be fixed by the invariance with respect to unbroken supersymmetry. The pleasant feature of such an approach is that the fermions are "hidden" inside the covariant derivatives and determinant of the vielbein, making the whole action short, with the explicit geometric meaning of each term. In the present paper we review this procedure in applications to the actions of the superparticle in $D=3$ realizing $N=4 \cdot 2^{k} \rightarrow N=2 \cdot 2^{k}$ pattern of supersymmetry breaking, the action of superparticle in $D=5$ with the $N=16$ supersymmetry broken down to $N=8$ one, the on-shell component actions of $N=1, D=5$ supermembrane and its dual cousins and the component action of $N=1$ supermembrane in $D=4$. All these explicit actions confirm our conjecture about the structure of the component action. Finally, we briefly discuss some related questions and further possible applications of our method.

\section{Basics of the method}

In this section we present main features of the coset approach, applying to supersymmetric models in which one half of the global supersymmetries are spontaneously broken. Before going to supersymmetric systems, we will consider how this method works in the purely bosonic case.

Let us split the generators of the target space of the $D$-dimensional Poincaré group, which is supposed to be spontaneously broken on the world volume down to the $d$-dimensional Poincaré subgroup, into the generators of unbroken $\{P, M, N\}$ and spontaneously broken $\{Z, K\}$ symmetries. The generators $P$ and $Z$ form $D$-dimensional translations, $M$ generators span the $s o(1, d-1)$ - Lorentz algebra on the world volume, the generators $N$ rotate broken translations $Z$ among themselves and thus they span $s o(D-d)$ algebra, while generators $K$ belong to the coset $s o(1, D-1) / s o(1, d-1) \times s o(D-d)$. All transformations of the $D$-dimensional Poincaré group can be realized by the left action of different group elements on the coset space 1

$$
g=e^{x P} e^{q(x) Z} e^{\Lambda(x) K} .
$$

${ }^{1}$ For the sake of brevity we suppress here all space-time indices. 
The spontaneous breaking of $Z$ and $K$ symmetries is reflected in the character of corresponding coset coordinates which are Goldstone fields $q(x)$ and $\Lambda(x)$ in the present case. The transformation properties of coordinates $x$ and fields $\{q(x), \Lambda(x)\}$ may be easily found in this approach, while all needed information about the geometry of the coset space (2.1) is contained in the Cartan forms

$$
g^{-1} d g=\Omega_{P} P+\Omega_{M} M+\Omega_{Z} Z+\Omega_{K} K+\Omega_{N} N .
$$

All Cartan forms except for $\Omega_{M}$ and $\Omega_{N}$ are transformed homogeneously under all symmetries. Due to the general theorem [13] not all of the above Goldstone fields have to be treated as independent. In the present case the fields $\Lambda(x)$ can be covariantly expressed through $x$-derivatives of $q(x)$ by imposing the constraint

$$
\Omega_{Z}=0 .
$$

Equations encoded in the conditions (2.3), do not contain dynamic restrictions and are purely kinematic. Thus, we are dealing with the fields $q(x)$ only. It is very important that the form $\Omega_{P}$ defines the vielbein $E$ ( $d$-bein in the present case), connecting the covariant world volume coordinate differentials $\Omega_{P}$ and the world volume coordinate differential $d x$ as

$$
\Omega_{P}=E \cdot d x .
$$

Combining all these ingredients, one may immediately write the action

$$
S=-\int d^{d} x+\int d^{d} x \operatorname{det} E,
$$

which is invariant under all symmetries. In (2.5) we have added the trivial first term to fulfill the condition $S_{q=0}=0$. The action (2.5) is just the static gauge form of the action of $p=(d-1)$-branes.

The supersymmetric generalization of the coset approach involves into the game new spinor generators $Q$ and $S$ which extend the $D$-dimensional Poincaré group to the supersymmetric one

$$
\{Q, Q\} \sim P, \quad\{S, S\} \sim P, \quad\{Q, S\} \sim Z
$$

The most interesting cases are those when the $Q$ supersymmetry is kept unbroken, while the $S$ supersymmetry is supposed to be spontaneously broken2. When $\# Q=\# S$ we are facing the so-called 1/2 Partial Breaking of Global Supersymmetry cases (PBGS), which most of all interesting supersymmetric domain walls belong to. Only such cases of supersymmetry breaking will be considered in this paper.

Now, all symmetries can be realized by group elements acting on the coset element

$$
g=e^{x P} e^{\theta Q} e^{\boldsymbol{q}(x, \theta) Z} e^{\boldsymbol{\psi}(x, \theta) S} e^{\boldsymbol{\Lambda}(x, \theta) K} .
$$

The main novel feature of the supersymmetric coset (2.7) is the appearance of the Goldstone superfields $\{\boldsymbol{q}(x, \theta)$, $\boldsymbol{\psi}(x, \theta), \boldsymbol{\Lambda}(x, \theta)\}$ which depend on the coordinates of the world volume superspace $\{x, \theta\}$. The rest of the coset approach machinery works in the same manner: one may construct the Cartan forms (2.2) for the coset (2.7)) (which will contain the new forms $\Omega_{Q}$ and $\Omega_{S}$ ), one may find the supersymmetric $d$-bein and corresponding bosonic $\nabla_{P}$ and spinor $\nabla_{Q}$ covariant derivatives, etc. One may even write the proper generalizations of the covariant constraints (2.3) as

$$
\Omega_{Z}=0, \quad \Omega_{S} \mid=0,
$$

where $\mid$ means the $d \theta$-projection of the form (see e.g. [10] and references therein). The $d \theta$-parts of these constraints are closely related with the "geometro-dynamical" constraint of the superembedding approach (see e.g. [22]).

Unfortunately, this similarity between purely bosonic and supersymmetric cases is not complete due to the existence of the following important new features of theories with partial breaking of global supersymmetry:

\footnotetext{
${ }^{2}$ If all supersymmetries are considered as spontaneously broken, the corresponding action can be constructed similarly to the bosonic case, resulting in the some synthesis of Volkov-Akulov 3] and Nambu -Goto actions. An enlightening example of such a construction can be found in 20 .
} 
- In contrast with the bosonic case, not all of the physical fields appear among the parameters of the coset. A famous example comes from the supersymmetric space-filling D3-brane (aka $N=1$ Born-Infeld theory) where the coset element (2.7) contains only $P, Q$ and $S$ generators [5, 7, while the field strength $F$ is "hidden" inside the superfield $\boldsymbol{\psi}: F \sim \nabla_{Q} \boldsymbol{\psi} \mid$. Nevertheless, it is true that the all physical bosonic components can be found in the quantity $\nabla_{Q} \psi \mid$.

- The supersymmetric generalization (2.8) of the bosonic kinematic constraints (2.3) in most cases contains not only kinematic conditions, but also dynamic superfield equations of motion. A prominent example again may be found in [5]. Moreover, in many cases it is unknown how to split these constraints into kinematical and dynamical ones.

- But the most unpleasant feature of the supersymmetric cases is that the standard methods of nonlinear realizations fail to construct the superfield action! The main reason for this is simple: all that we have at hands are the covariant Cartan forms, which we can construct the superfield invariants from, while the superspace Lagrangian is not invariant. Instead it is shifted by the full spinor derivatives under unbroken and/or broken supersymmetries.

Nevertheless, we are going to apply a coset approach to the supersymmetric cases and to demonstrate how on-shell component actions can be constructed within it. The main idea is to start with the Ansatz for the action manifestly invariant with respect to spontaneously broken supersymmetry. Funny enough, it is rather easy to do, due to the following properties:

- In our parametrization of the coset element (2.7) the superspace coordinates $\theta$ do not transform under broken supersymmetry. Thus, all components of superfields transform independently,

- The covariant derivatives $\nabla_{P}$ and $\nabla_{Q}$ are invariant under broken supersymmetry. Therefore, the bosonic physical components which are contained in $\nabla_{Q} \psi(x, \theta) \mid$ can be treated as "matter fields" (together with the field $\boldsymbol{q}(x, \theta) \mid$ itself $)$ with respect to broken supersymmetry,

- All physical fermionic components are just $\theta=0$ projections of the superfield $\boldsymbol{\psi}(x, \theta)$ and these components transform as the fermions of the Volkov-Akulov model [3] with respect to broken supersymmetry.

The immediate consequence of these facts is the conclusion that the physical fermionic components can enter the component on-shell action either through the determinant of the $d$-bein constructed with the help of the Cartan form $\Omega_{P}$ in the limit $\theta=0$, namely, $\mathcal{E}=E \mid$, through the space-time derivatives of the "matter fields" $\nabla_{P} \boldsymbol{q} \mid$, or through the Wess-Zumino terms if they exist. Thus, the most general Ansatz for the on-shell component action, which is invariant with respect to spontaneously broken supersymmetry, has the form

$$
S=\int d^{d} x-\int d^{d} x \operatorname{det} \mathcal{E} \mathcal{F}\left(\nabla_{Q} \boldsymbol{\psi}\left|, \nabla_{P} \boldsymbol{q}\right|\right)+S_{W Z}
$$

Note, that the arguments of the function $\mathcal{F}$ are the bosonic physical components $\nabla_{Q} \psi \mid$ and the covariant spacetime derivatives of $\boldsymbol{q}$ (which, by the way, are also contained in $\nabla_{Q} \boldsymbol{\psi} \mid$ ). In certain cases, for fixing an explicit form of the function $\mathcal{F}$ it is sufficient that the following two conditions be satisfied

1. The action (2.9) should have a proper bosonic limit, which is known in almost all interesting cases. One should note, that this limit for the action (2.9) is trivial

$$
S_{\text {bos }}=\int d^{d} x\left(1-\mathcal{F}\left(\nabla_{Q} \psi \mid, \partial_{P} q\right)\right) .
$$

2. The action (2.9) in the linear limit should possess a linear version of unbroken supersymmetry, i.e. it should be just the sum of the kinetic terms for all bosonic and fermionic components with the relative coefficients fixed by unbroken supersymmetry. 
One should note that the Wess-Zimino action, which is invariant under broken supersymmetry, can be also constructed from the Cartan forms following the recipe of ref. [21]. Thus, the role of unbroken supersymmetry is to fix the coefficients in the action (2.9) to achieve its invariance with respect to unbroken sypersymmetry.

In the next two sections of the present paper we will show how the coset approach works in the cases of the superparticle in $D=3$ and $D=5$ with the chiral and quartet Goldstone supermultiplets, respectively. Then in section 5 we will extend our analysis to the cases of $N=1$ supermembrane in $D=4$ as well as of the dual system $-N=1$ supersymmetric space filling D2-brane. In section 6 we will show that in order to construct $N=2$ supersymmetric action for the supermembrane action in $D=4$, one needs to add the corresponding Wess-Zumino term. In Appendices we collect the technical details, notation and explicit proof of invariance of the supermembrane action with respect to both, broken and unbroken supersymmetries. We conclude with some comments and perspectives.

\section{Superparticle in $\mathrm{D}=3$}

The main goal of this section is to provide the detailed structure of the component on-shell actions for the onedimensional system realizing a one half breaking of the global supersymmetry. As an example, we consider a system with $N=16 \rightarrow N=8$ pattern of supersymmetry breaking based on the superalgebra with two "semicentral charges" $(Z, \bar{Z})$. We show that the resulting component action describes a superparticle in $D=3$.

\subsection{Superparticle in $\mathrm{D}=3$ : kinematics}

It is a well known fact that the action for the given pattern of the supersymmetry breaking is completely defined by the choice of the corresponding Goldstone supermultiplet [4, 5, 6, 7, 8, 9, 10, 11, 12. The bosonic scalars of the supermultiplet are associated with the "semi-central charges" in the supersymmetry algebra (2.6). To describe a system with one complex boson (or two real bosons) one has to choose $N=16, d=1$ Poincaré superalgebra with two "semi-central charges" $(Z, \bar{Z})$

$$
\left\{Q^{i a}, \bar{Q}_{j b}\right\}=2 \delta_{b}^{a} \delta_{j}^{i} P, \quad\left\{S^{i a}, \bar{S}_{j b}\right\}=2 \delta_{b}^{a} \delta_{j}^{i} P, \quad\left\{Q^{i a}, S^{j b}\right\}=2 i \varepsilon^{a b} \varepsilon^{i j} Z, \quad\left\{\bar{Q}_{i a}, \bar{S}_{j b}\right\}=-2 i \varepsilon_{a b} \varepsilon_{i j} \bar{Z} .
$$

Here $i, a=1,2$ refer to the indices of the fundamental representations of two commuting $S U(2)$ groups. In (3.1) $P$ is the generator of one-dimensional translation, while $Q^{i a}, \bar{Q}_{i a}$ and $S^{i a}, \bar{S}_{i a}$ are the generators of unbroken and spontaneously broken $N=8$ supersymmetries, respectively. As we already explain in the Introduction, in the coset approach the statement that $S$ supersymmetry and $(Z, \bar{Z})$ translations are spontaneously broken is reflected in the structure of the element of the coset space

$$
g=e^{i t P} e^{\theta_{i a} Q^{i a}+\bar{\theta}^{i a} \bar{Q}_{i a}} e^{i(\boldsymbol{q} Z+\overline{\boldsymbol{q}} \bar{Z})} e^{\boldsymbol{\psi}_{i a} S^{i a}+\overline{\boldsymbol{\psi}}^{i a} \bar{S}_{i a} .}
$$

Once we state that the coordinates $\boldsymbol{\psi}$ and $\boldsymbol{q}$ are the superfields depending on the $N=8, d=1$ superspace coordinates $(t, \theta, \bar{\theta})$, then we are dealing with the spontaneously breaking of the corresponding symmetries. Thus, in our case we will treat $\boldsymbol{\psi}(t, \theta, \bar{\theta}), \boldsymbol{q}(t, \theta, \bar{\theta})$ as $N=8, d=1$ Goldstone superfields accompanying $N=16 \rightarrow N=8$ breaking of supersymmetry in one dimension.

The transformation properties of coordinates and superfields under both unbroken and broken supersymmetries are induced by the left multiplications of the group element $g_{0}$ on the coset (3.2)

$$
g_{0} g=g^{\prime} .
$$

Thus, for the unbroken supersymmetry with $g_{0}=e^{\varepsilon_{i a} Q^{i a}+\bar{\varepsilon}^{i a} \bar{Q}_{i a}}$ one gets

$$
\delta_{Q} t=i\left(\varepsilon_{i a} \bar{\theta}^{i a}+\bar{\varepsilon}^{i a} \theta_{i a}\right), \quad \delta_{Q} \theta_{i a}=\varepsilon_{i a}, \quad \delta_{Q} \bar{\theta}^{i a}=\bar{\varepsilon}^{i a},
$$

while for the broken supersymmetry with $g_{0}=e^{\eta_{i a} S^{i a}+\bar{\eta}^{i a} \bar{S}_{i a}}$ the transformations read

$$
\delta_{S} t=i\left(\eta_{i a} \overline{\boldsymbol{\psi}}^{i a}+\bar{\eta}^{i a} \boldsymbol{\psi}_{i a}\right), \quad \delta_{S} \boldsymbol{\psi}_{i a}=\eta_{i a}, \quad \delta_{S} \overline{\boldsymbol{\psi}}^{i a}=\bar{\eta}^{i a}, \quad \delta_{S} \boldsymbol{q}=-2 \eta_{i a} \theta^{i a}, \quad \delta_{S} \overline{\boldsymbol{q}}=2 \bar{\eta}^{i a} \bar{\theta}_{i a} .
$$


The local geometric properties of the system are specified by the left-invariant Cartan forms

$$
g^{-1} d g=i \omega_{P} P+\left(\omega_{Q}\right)_{i a} Q^{i a}+\left(\bar{\omega}_{Q}\right)^{i a} \bar{Q}_{i a}+i \omega_{Z} Z+i \bar{\omega}_{Z} \bar{Z}+\left(\omega_{S}\right)_{i a} S^{i a}+\left(\bar{\omega}_{S}\right)^{i a} \bar{S}_{i a}
$$

which can be explicitly written in the considered case as

$$
\begin{aligned}
& \omega_{P}=d t-i\left(\bar{\theta}^{i a} d \theta_{i a}+\theta_{i a} d \bar{\theta}^{i a}+\overline{\boldsymbol{\psi}}^{i a} d \boldsymbol{\psi}_{i a}+\boldsymbol{\psi}_{i a} d \overline{\boldsymbol{\psi}}^{i a}\right), \quad\left(\omega_{Q}\right)_{i a}=d \theta_{i a}, \quad\left(\bar{\omega}_{Q}\right)^{i a}=d \bar{\theta}^{i a}, \\
& \left(\omega_{S}\right)_{i a}=d \boldsymbol{\psi}_{i a}, \quad\left(\bar{\omega}_{S}\right)^{i a}=d \overline{\boldsymbol{\psi}}^{i a}, \quad \omega_{Z}=d \boldsymbol{q}+2 \boldsymbol{\psi}^{i a} d \theta_{i a}, \quad \bar{\omega}_{Z}=d \overline{\boldsymbol{q}}-2 \overline{\boldsymbol{\psi}}_{i a} d \bar{\theta}^{i a} .
\end{aligned}
$$

Using the covariant differentials $\left(\omega_{P}, d \theta_{i a}, d \bar{\theta}^{i a}\right)$ (3.6), one may construct the covariant derivatives

$$
\begin{aligned}
& \partial_{t}=E \nabla_{t}, \quad E=1-i\left(\boldsymbol{\psi}_{i a} \dot{\overline{\boldsymbol{\psi}}}^{i a}+\overline{\boldsymbol{\psi}}^{i a} \dot{\boldsymbol{\psi}}_{i a}\right), \quad E^{-1}=1+i\left(\boldsymbol{\psi}_{i a} \nabla_{t} \overline{\boldsymbol{\psi}}^{i a}+\overline{\boldsymbol{\psi}}^{i a} \nabla_{t} \boldsymbol{\psi}_{i a}\right), \\
& \nabla^{i a}=D^{i a}-i\left(\boldsymbol{\psi}_{k b} D^{i a} \overline{\boldsymbol{\psi}}^{k b}+\overline{\boldsymbol{\psi}}^{k b} D^{i a} \boldsymbol{\psi}_{k b}\right) \nabla_{t}=D^{i a}-i\left(\boldsymbol{\psi}_{k b} \nabla^{i a} \overline{\boldsymbol{\psi}}^{k b}+\overline{\boldsymbol{\psi}}^{k b} \nabla^{i a} \boldsymbol{\psi}_{k b}\right) \partial_{t}, \\
& \bar{\nabla}_{i a}=\bar{D}_{i a}-i\left(\boldsymbol{\psi}_{k b} \bar{D}_{i a} \overline{\boldsymbol{\psi}}^{k b}+\overline{\boldsymbol{\psi}}^{k b} \bar{D}_{i a} \boldsymbol{\psi}_{k b}\right) \nabla_{t}=\bar{D}_{i a}-i\left(\boldsymbol{\psi}_{k b} \bar{\nabla}_{i a} \overline{\boldsymbol{\psi}}^{k b}+\overline{\boldsymbol{\psi}}^{k b} \bar{\nabla}_{i a} \boldsymbol{\psi}_{k b}\right) \partial_{t},
\end{aligned}
$$

where

$$
D^{i a}=\frac{\partial}{\partial \theta_{i a}}-i \bar{\theta}^{i a} \partial_{t}, \quad \bar{D}_{i a}=\frac{\partial}{\partial \bar{\theta}^{i a}}-i \theta_{i a} \partial_{t}, \quad\left\{D^{i a}, \bar{D}_{j b}\right\}=-2 i \delta_{b}^{a} \delta_{j}^{i} \partial_{t} .
$$

The covariant derivatives (3.7) satisfy the following (anti)commutation relations

$$
\begin{aligned}
& \left\{\nabla^{i a}, \nabla^{j b}\right\}=-2 i\left(\nabla^{i a} \boldsymbol{\psi}_{k c} \nabla^{j b} \overline{\boldsymbol{\psi}}^{k c}+\nabla^{i a} \overline{\boldsymbol{\psi}}^{k c} \nabla^{j b} \boldsymbol{\psi}_{k c}\right) \nabla_{t}, \\
& \left\{\bar{\nabla}_{i a}, \bar{\nabla}_{j b}\right\}=-2 i\left(\bar{\nabla}_{i a} \boldsymbol{\psi}_{k c} \bar{\nabla}_{j b} \overline{\boldsymbol{\psi}}^{k c}+\bar{\nabla}_{i a} \overline{\boldsymbol{\psi}}^{k c} \bar{\nabla}_{j b} \boldsymbol{\psi}_{k c}\right) \nabla_{t}, \\
& {\left[\nabla_{t}, \nabla^{i a}\right]=-2 i\left(\nabla_{t} \boldsymbol{\psi}_{k c} \nabla^{i a} \overline{\boldsymbol{\psi}}^{k c}+\nabla_{t} \overline{\boldsymbol{\psi}}^{k c} \nabla^{i a} \boldsymbol{\psi}_{k c}\right) \nabla_{t},} \\
& {\left[\nabla_{t}, \bar{\nabla}_{i a}\right]=-2 i\left(\nabla_{t} \boldsymbol{\psi}_{k c} \bar{\nabla}_{i a} \overline{\boldsymbol{\psi}}^{k c}+\nabla_{t} \overline{\boldsymbol{\psi}}^{k c} \bar{\nabla}_{i a} \boldsymbol{\psi}_{k c}\right) \nabla_{t},} \\
& \left\{\nabla^{i a}, \bar{\nabla}_{j b}\right\}=-2 i \delta_{b}^{a} \delta_{j}^{i} \nabla_{t}-2 i\left(\nabla^{i a} \boldsymbol{\psi}_{k c} \bar{\nabla}_{j b} \overline{\boldsymbol{\psi}}^{k c}+\nabla^{i a} \overline{\boldsymbol{\psi}}^{k c} \bar{\nabla}_{j b} \boldsymbol{\psi}_{k c}\right) \nabla_{t} .
\end{aligned}
$$

To reduce the number of independent Goldstone superfields let us impose the conditions on the $d \theta$-projections of the Cartan forms $\left(\omega_{Z}, \bar{\omega}_{Z}\right)$ (3.6)

$$
\left\{\begin{array}{l}
\left.\omega_{Z}\right|_{\theta}=0, \\
\left.\bar{\omega}_{Z}\right|_{\theta}=0,
\end{array} \quad \Rightarrow \quad \begin{array}{ll}
\bar{\nabla}_{i a} \boldsymbol{q}=0, & \nabla^{i a} \boldsymbol{q}-2 \boldsymbol{\psi}^{i a}=0 \\
\nabla^{i a} \overline{\boldsymbol{q}}=0, & \bar{\nabla}_{i a} \overline{\boldsymbol{q}}+2 \overline{\boldsymbol{\psi}}_{i a}=0 .
\end{array}\right.
$$

One part of these kinematical constraints can be recognized as the covariant chirality conditions on the superfields $\boldsymbol{q}$ and $\overline{\boldsymbol{q}}$, while the remaining two equations express the fermionic Goldstone superfields $\boldsymbol{\psi}^{i a}$ and $\overline{\boldsymbol{\psi}}_{i a}$ as the spinor derivatives of the bosonic superfields $\boldsymbol{q}$ and $\overline{\boldsymbol{q}}$, thereby realizing the Inverse Higgs phenomenon [13].

\subsection{Superparticle in $\mathrm{D}=3$ : dynamics}

It is well known that the standard chirality conditions are not enough to select an irreducible $N=8, d=1$ supermultiplet: one has impose additional, second order in the spinor derivatives constraints on the superfield $\{\boldsymbol{q}, \overline{\boldsymbol{q}}\}$ 23]. Unfortunately, as it often happened in the coset approach, the direct covariantization of the irreducibility constraints is not covariant [5, while the simultaneous covariantization of the constraints and the equations of motion works perfectly. That is why we propose the following equations which should describe our superparticle

$$
\nabla^{i a} \boldsymbol{\psi}_{j b}=0, \quad \bar{\nabla}_{i a} \bar{\psi}^{j b}=0 .
$$

These equations are covariant with respect to both unbroken and broken supersymmetries. One should wonder whether the equations (3.11) are self-consistent? Indeed, due to eqs. (3.10) from (3.11) we have

$$
\nabla^{i a} \boldsymbol{\psi}_{j b}=\frac{1}{2} \nabla^{i a} \nabla_{j b} \boldsymbol{q}=0 \quad \Rightarrow \quad\left\{\nabla^{i a}, \nabla_{j b}\right\} \boldsymbol{q}=0 .
$$


So, one may expect some additional conditions on the superfield $\boldsymbol{q}$ due to the relations (3.9). However, on the constraints surface in (3.11) we have

$$
\left\{\nabla^{i a}, \nabla^{j b}\right\}=0, \quad\left\{\bar{\nabla}_{i a}, \bar{\nabla}_{j b}\right\}=0,
$$

and thus the equations (3.11) are perfectly self-consistent.

It is worth mentioning that the rest of the commutators in (3.9) are also simplified, when (3.11) are satisfied. Indeed, on the constraints (3.11) surface they read

$$
\left\{\nabla^{i a}, \bar{\nabla}_{j b}\right\}=-2 i \delta_{j}^{i} \delta_{b}^{a}(1+\boldsymbol{\lambda} \overline{\boldsymbol{\lambda}}) \nabla_{t}, \quad\left[\nabla_{t}, \nabla^{i a}\right]=2 i \overline{\boldsymbol{\lambda}} \nabla_{t} \boldsymbol{\psi}^{i a} \nabla_{t}, \quad\left[\nabla_{t}, \bar{\nabla}_{i a}\right]=2 i \boldsymbol{\lambda} \nabla_{t} \overline{\boldsymbol{\psi}}_{i a} \nabla_{t},
$$

where we introduced the superfields $\{\boldsymbol{\lambda}, \overline{\boldsymbol{\lambda}}\}$

$$
\left\{\begin{array} { l } 
{ \overline { \nabla } _ { i a } \boldsymbol { \psi } _ { j b } + \varepsilon _ { i j } \varepsilon _ { a b } \boldsymbol { \lambda } = 0 , } \\
{ \nabla ^ { i a } \overline { \boldsymbol { \psi } } ^ { j b } + \varepsilon ^ { i j } \varepsilon ^ { a b } \overline { \boldsymbol { \lambda } } = 0 , }
\end{array} \Rightarrow \left\{\begin{array}{c}
\nabla_{t} \boldsymbol{q}+\frac{i \boldsymbol{\lambda}}{1+\boldsymbol{\lambda} \boldsymbol{\lambda}}=0, \\
\nabla_{t} \overline{\boldsymbol{q}}-\frac{i \boldsymbol{\lambda}}{1+\boldsymbol{\lambda} \boldsymbol{\lambda}}=0 .
\end{array}\right.\right.
$$

The superfield equations (3.11) lead in the bosonic limit to the following equation of motion for the complex scalar field $q=\left.\boldsymbol{q}\right|_{\theta=0}$ :

$$
\frac{d}{d t}\left[\frac{\dot{q}}{\sqrt{1-4 \dot{q} \dot{\bar{q}}}}\right]=0 .
$$

The last equation can be easy deduced from the bosonic action

$$
S_{\text {bos }}=\int d t(1-\sqrt{1-4 \dot{q}}) .
$$

Thus, the bosonic action for a particle in $D=3$ space-time is known.

\subsection{Superparticle in $\mathrm{D}=3$ : component action}

Despite the explicit construction of the proper equations of motion within the superfield version of the coset approach, it is poorly adapted for the construction of the action. That is why in the paper [17 the component version of the coset approach to construct the actions has been proposed. In the application to the present case, the basic steps of this method can be formulated as follows:

- Firstly, on-shell our $N=8$ supermultiplet $\{\boldsymbol{q}, \overline{\boldsymbol{q}}\}$ contains the following physical components:

$$
q=\left.\boldsymbol{q}\right|_{\theta=0}, \quad \bar{q}=\left.\overline{\boldsymbol{q}}\right|_{\theta=0}, \quad \psi_{i a}=\left.\boldsymbol{\psi}_{i a}\right|_{\theta=0}, \quad \bar{\psi}^{i a}=\left.\overline{\boldsymbol{\psi}}^{i a}\right|_{\theta=0} .
$$

They are just the first components of the superfields parameterizing the coset (3.2).

- Secondly, with respect to broken supersymmetry $\delta \theta=\delta \bar{\theta}=0$ (3.4). This means, that the transformation properties of the physical components $\left\{q, \bar{q}, \psi_{i a}, \bar{\psi}^{i a}\right\}$ under broken supersymmetry can be extracted from the coset

$$
\left.g\right|_{\theta=0}=e^{i t P} e^{i(q Z+\bar{q} \bar{Z})} e^{\psi_{i a} S^{i a}+\bar{\psi}^{i a} \bar{S}_{i a}} .
$$

In other words, the fields $\left\{q, \bar{q}, \psi_{i a}, \bar{\psi}^{i a}\right\}$ parameterize the coset (3.18) which is responsible for full breaking of the $S$ supersymmetry. Moreover, with respect to this supersymmetry the fields $\{q, \bar{q}\}$ are just "matter fields", because $\delta_{S} q=\delta_{S} \bar{q}=0$, while the fermions $\left\{\psi_{i a}, \bar{\psi}^{i a}\right\}$ are just Goldstone fermions. This means that the component action has to be of the Volkov-Akulov type [3, i.e. the fermions $\left\{\psi_{i a}, \bar{\psi}^{i a}\right\}$ may enter the action through the einbein $\mathcal{E}$ or through the covariant derivatives $\mathcal{D}_{t} q, \mathcal{D}_{t} \bar{q}$ only, with

$$
\partial_{t}=\mathcal{E} \mathcal{D}_{t}, \quad \mathcal{E}=\left.E\right|_{\theta=0}=1-i\left(\psi_{i a} \dot{\bar{\psi}}^{i a}+\bar{\psi}^{i a} \dot{\psi}_{i a}\right), \quad \mathcal{E}^{-1}=1+i\left(\psi_{i a} \mathcal{D}_{t} \bar{\psi}^{i a}+\bar{\psi}^{i a} \mathcal{D}_{t} \psi_{i a}\right)
$$

Thus, the unique candidate to be the component on-shell action, invariant with respect to spontaneously broken $S$ supersymmetry reads

$$
S=\alpha \int d t+\int d t \mathcal{E F}\left[\mathcal{D}_{t} q \mathcal{D}_{t} \bar{q}\right]
$$

with an arbitrary, for the time being, function $\mathcal{F}$ and a constant parameter $\alpha$. 
- Finally, considering the bosonic limit of the action (3.20) and comparing it with the known bosonic action (3.17) one may find the function $\mathcal{F}$ :

$$
\int d t(\alpha+\mathcal{F}[\dot{q} \dot{\bar{q}}])=\int d t(1-\sqrt{1-4 \dot{q}} \dot{\bar{q}}) \Rightarrow \mathcal{F}=(1-\alpha-\sqrt{1-4 \dot{q} \dot{\bar{q}}}) .
$$

Therefore, the most general component action possessing the proper bosonic limit (3.17) and invariant under spontaneously broken supersymmetry has the form

$$
S=\alpha \int d t+(1-\alpha) \int d t \mathcal{E}-\int d t \mathcal{E} \sqrt{1-4 \mathcal{D}_{t} q \mathcal{D}_{t} \bar{q}} .
$$

In principle, the invariance of the action (3.22) under broken supersymmetry is evident. Nevertheless, it should be explicitly checked.

From (3.4) we obtain the total variations of our components and the time coordinate $t$ :

$$
\delta_{S} t=i\left(\eta_{i a} \bar{\psi}^{i a}+\bar{\eta}^{i a} \psi_{i a}\right), \quad \delta_{S} \psi_{i a}=\eta_{i a}, \quad \delta_{S} \bar{\psi}^{i a}=\bar{\eta}^{i a}, \quad \delta_{S} q=0, \quad \delta_{S} \bar{q}=0 .
$$

Therefore, the transformations of the components in the fixed point read

$$
\delta_{S}^{*} q=\delta_{S} q-\delta_{S} t \dot{q}, \quad \delta_{S}^{*} \psi_{i a}=\delta_{S} \psi_{i a}-\delta_{S} t \dot{\psi_{i a}} .
$$

Then, it immediately follows from (3.24) and definitions (3.19) that

$$
\delta_{S}^{*}\left(\mathcal{E} \mathcal{F}\left[\mathcal{D}_{t} q \mathcal{D}_{t} \bar{q}\right]\right)=-i \partial_{t}\left[\left(\eta_{i a} \bar{\psi}^{i a}+\bar{\eta}^{i a} \psi_{i a}\right) \mathcal{E} \mathcal{F}\left[\mathcal{D}_{t} q \mathcal{D}_{t} \bar{q}\right]\right]
$$

Thus, the two last terms in the action (3.22) are invariant, while the invariance of the first term is evident.

The final step is to check the invariance of the action (3.22) under unbroken supersymmetry which is realized on the components as follows:

$$
\begin{aligned}
& \delta_{Q}^{*} q=-2 \varepsilon^{i a} \psi_{i a}+i\left(\varepsilon^{i a} \psi_{i a} \bar{\lambda}+\bar{\varepsilon}^{i a} \bar{\psi}_{i a} \lambda\right) \partial_{t} q \\
& \delta_{Q}^{*} \psi_{i a}=\bar{\varepsilon}_{i a} \lambda+i\left(\varepsilon^{j b} \psi_{j b} \bar{\lambda}+\bar{\varepsilon}^{j b} \bar{\psi}_{j b} \lambda\right) \partial_{t} \psi_{i a} .
\end{aligned}
$$

Here, $\lambda$ is the first component of the superfield $\boldsymbol{\lambda}$ defined in (3.15)

$$
\lambda=\frac{2 i \mathcal{D}_{t} q}{1+\sqrt{1-4 \mathcal{D}_{t} q \mathcal{D}_{t} \overline{\bar{q}}}} .
$$

From (3.26) and the definitions (3.19) one may easily find the transformation properties of the main ingredients

$$
\begin{aligned}
& \delta_{Q}^{*} \mathcal{E}=i \partial_{t}\left[\left(\varepsilon^{j b} \psi_{j b} \bar{\lambda}+\bar{\varepsilon}^{j b} \bar{\psi}_{j b} \lambda\right) \mathcal{E}\right]-2 i\left(\varepsilon^{j b} \dot{\psi}_{j b} \bar{\lambda}+\bar{\varepsilon}^{j b} \dot{\bar{\psi}}_{j b} \lambda\right) \\
& \delta_{Q}^{*} \mathcal{D}_{t} q=i\left(\varepsilon^{j b} \psi_{j b} \bar{\lambda}+\bar{\varepsilon}^{j b} \bar{\psi}_{j b} \lambda\right) \partial_{t}\left(\mathcal{D}_{t} q\right)-2 \varepsilon^{j b} \mathcal{D}_{t} \psi_{j b}+2 i\left(\varepsilon^{j b} \mathcal{D}_{t} \psi_{j b} \bar{\lambda}+\bar{\varepsilon}^{j b} \mathcal{D}_{t} \bar{\psi}_{j b} \lambda\right) \mathcal{D}_{t} q .
\end{aligned}
$$

Now, one may calculate the variation of the integrand in the action (3.20)

$$
\begin{aligned}
& \delta_{Q}^{*}(\mathcal{E} \mathcal{F})=2 \partial_{t}\left[\mathcal{E} \frac{\varepsilon^{j b} \psi_{j b} \mathcal{D}_{t} \bar{q}-\bar{\varepsilon}^{j b} \bar{\psi}_{j b} \mathcal{D}_{t} q}{1+\sqrt{1-4 \mathcal{D}_{t} q \mathcal{D}_{t} \bar{q}}} \mathcal{F}\right]+ \\
& +\frac{\varepsilon^{j b} \dot{\psi}_{j b} \mathcal{D}_{t} \bar{q}-\bar{\varepsilon}^{j b} \dot{\bar{\psi}}_{j b} \mathcal{D}_{t} q}{1+\sqrt{1-4 \mathcal{D}_{t} q \mathcal{D}_{t} \bar{q}}}\left[-4 \mathcal{F}-2 \mathcal{F}^{\prime}\left(1+\sqrt{1-4 \mathcal{D}_{t} q \mathcal{D}_{t} \bar{q}}-4 \mathcal{D}_{t} q \mathcal{D}_{t} \bar{q}\right)\right] .
\end{aligned}
$$

Substituting the function $\mathcal{F}$ (3.21) and its derivative over its argument $\mathcal{D}_{t} q \mathcal{D}_{t} \bar{q}$, we find that the second term in the variation (3.29) cancels out, provided $\alpha=2$. Keeping in mind that the first term in the action (3.22) is trivially invariant under unbroken supersymmetry, we conclude that the unique component action invariant under both unbroken and broken $N=8$ supersymmetries reads

$$
S=2 \int d t-\int d t \mathcal{E}\left(1+\sqrt{1-4 \mathcal{D}_{t} q \mathcal{D}_{t} \bar{q}}\right)
$$

We end this section with two comments.

Firstly, one should note that the construction of the component action, we considered in the previous section, has two interesting peculiarities: 
- It is based on the coset realization of the $N=16$ superalgebra (3.1)

- In the component action (3.30) the summation over indices $\{i, a\}$ of two $S U(2)$ groups affected only physical fermions $\left\{\psi_{i a}, \bar{\psi}^{i a}\right\}$.

It is quite clear, that in such a situation one may consider two subalgebras of $N=16$ superalgebra:

- $N=8$ supersymmetry, by choosing the corresponding supercharges as

$$
\tilde{Q}^{i} \equiv Q^{i 1}, \quad \widetilde{\bar{Q}}_{i} \equiv \bar{Q}_{i 1}, \quad \tilde{S}^{i} \equiv S^{i 2}, \quad \widetilde{\bar{S}}_{i} \equiv \bar{S}_{i 2},
$$

- $N=4$ supersymmetry with the supercharges

$$
\hat{Q} \equiv Q^{11}, \quad \widehat{\bar{Q}} \equiv \bar{Q}_{11}, \quad \hat{S} \equiv S^{22}, \quad \widehat{\bar{S}} \equiv \bar{S}_{22} .
$$

It is evident that the corresponding component actions will be given by the same expression (3.30), in which the "new" einbeins and covariant derivatives read

$$
\begin{aligned}
& N=8 \text { case: }\left\{\partial_{t}=\tilde{\mathcal{E}} \tilde{\mathcal{D}}_{t}, \quad \tilde{\mathcal{E}}=1-i\left(\psi_{i 2} \dot{\bar{\psi}}^{i 2}+\bar{\psi}^{i 2} \dot{\psi}_{i 2}\right), \quad \tilde{\mathcal{E}}^{-1}=1+i\left(\psi_{i 2} \tilde{\mathcal{D}}_{t} \bar{\psi}^{i 2}+\bar{\psi}^{i 2} \tilde{\mathcal{D}}_{t} \psi_{i 2}\right),\right. \\
& N=4 \text { case: }\left\{\partial_{t}=\hat{\mathcal{E}} \hat{\mathcal{D}}_{t}, \quad \hat{\mathcal{E}}=1-i\left(\psi_{22} \dot{\bar{\psi}}^{22}+\bar{\psi}^{22} \dot{\psi}_{22}\right), \quad \hat{\mathcal{E}}^{-1}=1+i\left(\psi_{22} \hat{\mathcal{D}}_{t} \bar{\psi}^{22}+\bar{\psi}^{22} \hat{\mathcal{D}}_{t} \psi_{22}\right) .\right.
\end{aligned}
$$

Thus, we see that the action (3.30) has a universal character, describing the series of theories with the following patterns of global supersymmetry breaking $N=16 \rightarrow N=8, N=8 \rightarrow N=4$ and $N=4 \rightarrow N=2$.

Secondly, it is almost evident, that the universality of the action (3.30) can be used to extend our construction to the cases of $N=4 \cdot 2^{k}$ supersymmetries by adding the needed numbers of $S U(2)$ indices to the superscharges as

$$
Q \rightarrow Q^{\alpha_{1} \ldots \alpha_{k}}, \bar{Q} \rightarrow \bar{Q}_{\alpha_{1} \ldots \alpha_{k}}, \quad S \rightarrow S^{\alpha_{1} \ldots \alpha_{k}}, \bar{S} \rightarrow \bar{S}_{\alpha_{1} \ldots \alpha_{k}}
$$

obeying the $N=4 \cdot 2^{k}$ Poincaré superalgebra

$$
\begin{aligned}
& \left\{Q^{\alpha_{1} \ldots \alpha_{k}}, \bar{Q}_{\beta_{1} \ldots \beta_{k}}\right\}=2 \delta_{\beta_{1}}^{\alpha_{1}} \ldots \delta_{\beta_{k}}^{\alpha_{k}} P, \quad\left\{S^{\alpha_{1} \ldots \alpha_{k}}, \bar{S}_{\beta_{1} \ldots \beta_{k}}\right\}=2 \delta_{\beta_{1}}^{\alpha_{1}} \ldots \delta_{\beta_{k}}^{\alpha_{k}} P, \\
& \left\{Q^{\alpha_{1} \ldots \alpha_{k}}, S^{\beta_{1} \ldots \beta_{k}}\right\}=2 i \varepsilon^{\alpha_{1} \beta_{1}} \ldots \varepsilon^{\alpha_{k} \beta_{k}} Z, \quad\left\{\bar{Q}_{\alpha_{1} \ldots \alpha_{k}}, \bar{S}_{\beta_{1} \ldots \beta_{k}}\right\}=-2 i \varepsilon_{\alpha_{1} \beta_{1}} \ldots \varepsilon_{\alpha_{k} \beta_{k}} \bar{Z}, .
\end{aligned}
$$

Once again, the component action describing superparticles in $D=3$ space with $N=4 \cdot 2^{k}$ Poincaré supersymmetry partially broken down to the $N=2 \cdot 2^{k}$ one will be given by the same expression (3.30) with the following substitutions

$$
\psi \rightarrow \psi_{\alpha_{1} \ldots \alpha_{k}}, \bar{\psi} \rightarrow \bar{\psi}^{\alpha_{1} \ldots \alpha_{k}}, \mathcal{E}=1-i\left(\psi_{\alpha_{1} \ldots \alpha_{k}} \dot{\bar{\psi}}^{\alpha_{1} \ldots \alpha_{k}}+\bar{\psi}^{\alpha_{1} \ldots \alpha_{k}} \dot{\psi}_{\alpha_{1} \ldots \alpha_{k}}\right)
$$

\section{Superparticle in $\mathrm{D}=5$}

In this section we will apply our approach to $N=16$ superparticle in $D=5$. The corresponding superfield equations of motion for this system, which possesses 8 manifest and 8 spontaneously broken supersymmetries, have been constructed within the coset approach in [11, while the action is still unknown.

To describe the superparticle in $D=5$ with 16 supersymmetries one has to start with the following superalgebra

$$
\left\{Q_{\alpha}^{i}, Q_{\beta}^{j}\right\}=\varepsilon^{i j} \Omega_{\alpha \beta} P, \quad\left\{Q_{\alpha}^{i}, S^{b \beta}\right\}=\delta_{\alpha}^{\beta} Z^{i b}, \quad\left\{S^{a \alpha}, S^{b \beta}\right\}=-\varepsilon^{a b} \Omega^{\alpha \beta} P, \quad(i, a=1,2 ; \alpha, \beta=1,2,3,4)
$$

where the invariant $\operatorname{Spin}(5)$ symplectic metric $\Omega_{\alpha \beta}$, allowing to raise and lower the spinor indices, obeys the conditions 3

$$
\Omega_{\alpha \beta}=-\Omega_{\beta \alpha}, \quad \Omega^{\alpha \beta}=-\frac{1}{2} \varepsilon^{\alpha \beta \lambda \sigma} \Omega_{\lambda \sigma}, \quad \Omega_{\alpha \beta}=-\frac{1}{2} \varepsilon_{\alpha \beta \lambda \sigma} \Omega^{\lambda \sigma}, \quad \Omega_{\alpha \beta} \Omega^{\beta \gamma}=\delta_{\alpha}^{\gamma} .
$$

\footnotetext{
${ }^{3}$ We use the following convention: $\varepsilon^{\alpha \beta \lambda \sigma} \varepsilon_{\alpha \beta \lambda \sigma}=24, \varepsilon^{\alpha \beta \lambda \sigma} \varepsilon_{\alpha \beta \mu \rho}=2\left(\delta_{\mu}^{\lambda} \delta_{\rho}^{\sigma}-\delta_{\rho}^{\lambda} \delta_{\mu}^{\sigma}\right)$.
} 
From the one-dimensional perspective this algebra is $N=16$ super Poincaré algebra with four central charges $Z^{i a}$. If we are going to treat $S$ supersymmetry to be spontaneously broken, then we have to consider the following element of the coset 4

$$
g=e^{t P} e^{\theta_{i}^{\alpha} Q_{\alpha}^{i}} e^{\boldsymbol{q}_{i a} Z^{i a}} e^{\boldsymbol{\psi}_{a \alpha} S^{a \alpha}}
$$

Here $\left(t, \theta_{i}^{\alpha}\right)$ are the coordinates of $N=8, d=1$ superspace while $\boldsymbol{q}_{i a}=\boldsymbol{q}_{i a}\left(t, \theta_{i}^{\alpha}\right), \boldsymbol{\psi}_{a \alpha}=\boldsymbol{\psi}_{a \alpha}\left(t, \theta_{i}^{\alpha}\right)$, are the Goldstone superfields.

Similarly to the case considered in the previous section, one may find the transformation properties of the coordinates and superfields, by acting from the left on the coset element (4.3) by different elements of the group with constant parameters. So, for the unbroken supersymmetry $\left(g_{0}=\exp \left(\varepsilon_{i}^{\alpha} Q_{\alpha}^{i}\right)\right)$ one gets

$$
\delta_{Q} t=-\frac{1}{2} \varepsilon_{i}^{\alpha} \theta^{i \beta} \Omega_{\alpha \beta}, \quad \delta_{Q} \theta_{i}^{\alpha}=\varepsilon_{i}^{\alpha}
$$

while for the broken supersymmetry $\left(g_{0}=\exp \left(\eta_{a \alpha} S^{a \alpha}\right)\right)$ the corresponding transformations read

$$
\delta_{S} t=-\frac{1}{2} \eta_{\alpha}^{a} \boldsymbol{\psi}_{a \beta} \Omega^{\alpha \beta}, \quad \delta_{S} \boldsymbol{\psi}_{a \alpha}=\eta_{a \alpha}, \quad \delta_{S} \boldsymbol{q}_{i a}=-\eta_{a \alpha} \theta_{i}^{\alpha}
$$

The last needed ingredient is the Cartan forms, defined in a standard way as

$$
g^{-1} d g=\omega_{P} P+\left(\omega_{Q}\right)_{i}^{\alpha} Q_{\alpha}^{i}+\left(\omega_{Z}\right)_{i a} Z^{i a}+\left(\omega_{S}\right)_{a \alpha} S^{a \alpha}
$$

with

$$
\begin{aligned}
& \omega_{P}=d t-\frac{1}{2} d \theta_{i}^{\alpha} \theta^{i \beta} \Omega_{\alpha \beta}+\frac{1}{2} d \boldsymbol{\psi}_{a \alpha} \boldsymbol{\psi}_{\beta}^{a} \Omega^{\alpha \beta}, \quad\left(\omega_{Z}\right)_{i a}=d \boldsymbol{q}_{i a}-d \theta_{i}^{\alpha} \boldsymbol{\psi}_{a \alpha} \\
& \left(\omega_{Q}\right)_{i}^{\alpha}=d \theta_{i}^{\alpha}, \quad\left(\omega_{S}\right)_{a \alpha}=d \boldsymbol{\psi}_{a \alpha} .
\end{aligned}
$$

Using the covariant differentials $\left\{\omega_{P},\left(\omega_{Q}\right)_{i}^{\alpha}\right\}$ one may construct the covariant derivatives $\nabla_{t}$ and $\nabla_{\alpha}^{i}$

$$
\begin{aligned}
\partial_{t} & =E \nabla_{t}, \quad E=1+\frac{1}{2} \Omega^{\beta \gamma} \boldsymbol{\psi}_{\beta}^{a} \partial_{t} \boldsymbol{\psi}_{a \gamma}, \quad E^{-1}=1-\frac{1}{2} \Omega^{\beta \gamma} \boldsymbol{\psi}_{\beta}^{a} \nabla_{t} \boldsymbol{\psi}_{a \gamma}, \\
\nabla_{\alpha}^{i} & =D_{\alpha}^{i}+\frac{1}{2} \Omega^{\beta \gamma} \boldsymbol{\psi}_{\beta}^{a} D_{\alpha}^{i} \boldsymbol{\psi}_{a \gamma} \nabla_{t}=D_{\alpha}^{i}+\frac{1}{2} \Omega^{\beta \gamma} \boldsymbol{\psi}_{\beta}^{a} \nabla_{\alpha}^{i} \boldsymbol{\psi}_{a \gamma} \partial_{t},
\end{aligned}
$$

where

$$
D_{\alpha}^{i}=\frac{\partial}{\partial \theta_{i}^{\alpha}}+\frac{1}{2} \theta^{i \beta} \Omega_{\alpha \beta} \partial_{t}, \quad\left\{D_{\alpha}^{i}, D_{\beta}^{j}\right\}=\varepsilon^{i j} \Omega_{\alpha \beta} \partial_{t} .
$$

These covariant derivatives satisfy the following (anti)commutation relations:

$$
\begin{aligned}
& \left\{\nabla_{\alpha}^{i}, \nabla_{\beta}^{j}\right\}=\varepsilon^{i j} \Omega_{\alpha \beta} \nabla_{t}+\Omega^{\lambda \sigma} \nabla_{\alpha}^{i} \boldsymbol{\psi}_{\lambda}^{b} \nabla_{\beta}^{j} \boldsymbol{\psi}_{b \sigma} \nabla_{t}, \\
& {\left[\nabla_{t}, \nabla_{\alpha}^{i}\right]=\Omega^{\beta \gamma} \nabla_{t} \boldsymbol{\psi}_{\beta}^{b} \nabla_{\alpha}^{i} \boldsymbol{\psi}_{b \gamma} \nabla_{t} .}
\end{aligned}
$$

Now, in a full analogy with the previously considered case, we impose the following invariant condition on the $d \theta$-projections of Cartan form $\left(\omega_{Z}\right)_{i a}$ (4.7):

$$
\left.\left(\omega_{Z}\right)_{i a}\right|_{\theta}=0 \Rightarrow\left\{\begin{array}{l}
\nabla_{\alpha}^{(j} \boldsymbol{q}_{a}^{i)}=0 \\
\nabla_{\alpha}^{i} \boldsymbol{q}_{i a}-2 \boldsymbol{\psi}_{a \alpha}=0 .
\end{array}\right.
$$

The condition (4.12b) identifies the fermionic superfield $\boldsymbol{\psi}_{a \alpha}$ with the spinor derivatives of the superfield $\boldsymbol{q}_{i a}$, just reducing the independent superfields to bosonic $\boldsymbol{q}_{i a}$ ones (this is again the Inverse Higgs Phenomenon [13]). The

\footnotetext{
${ }^{4}$ Here, we strictly follow the notations adopted in [11] which are slightly different with those we used in the previous sections.
} 
conditions $\left(4.12 a\right.$ ) are more restrictive - they nullify all auxiliary components in the superfield $\boldsymbol{q}_{i a}$. Indeed, it immediately follows from (4.12) that

$$
\frac{3}{2} \nabla_{\beta}^{j} \boldsymbol{\psi}_{a \alpha}=\left\{\nabla_{\beta}^{j}, \nabla_{\alpha}^{i}\right\} \boldsymbol{q}_{i a}-\frac{1}{2}\left\{\nabla_{\alpha}^{j}, \nabla_{\beta}^{i}\right\} \boldsymbol{q}_{i a} .
$$

Using anti-commutators (4.11), one may solve this equation as follows:

$$
\nabla_{\beta}^{j} \boldsymbol{\psi}_{a \alpha}+\frac{1}{2} \lambda_{a}^{j} \Omega_{\alpha \beta}=0
$$

where the superfield $\boldsymbol{\lambda}^{i a}$ is defined as $\left(\boldsymbol{\lambda}^{2}=\boldsymbol{\lambda}^{i a} \boldsymbol{\lambda}_{i a}\right)$

$$
\nabla_{t} \boldsymbol{q}^{i a}-\frac{1}{2} \frac{\lambda^{i a}}{1+\frac{\boldsymbol{\lambda}^{2}}{8}}=0 .
$$

Thus, we have the on-shell situation. In [1] the corresponding bosonic equation of motion has been found to be

$$
\frac{d}{d t}\left(\frac{\dot{q}_{i a}}{\sqrt{1-2 \dot{q}^{j b} \dot{q}_{j b}}}\right)=0,
$$

where $q_{i a}=\left.\boldsymbol{q}_{i a}\right|_{\theta=0}$ are the first components of the superfield $\boldsymbol{q}_{i a}$. The equation of motion (4.14) corresponds to the static-gauge form of Nambu-Goto action for the massive particle in $D=5$ space-time

$$
S_{b o s} \sim \int d t\left(1-\sqrt{1-2 \dot{q}^{i a} \dot{q}_{i a}}\right) .
$$

To construct the on-shell component action we will follow the same procedure which was described above in full details. So, we will omit unessential details concentrating only on the new features.

If we are interested in the invariance with respect to broken $S$ supersymmetry, then we may consider the reduced coset element

$$
\left.g\right|_{\theta=0}=e^{t P} e^{q_{i a} Z^{i a}} e^{\psi_{a \alpha} S^{a \alpha}} .
$$

Here, $q_{i a}$ and $\psi_{a \alpha}$ are the first components of the superfields $\boldsymbol{q}_{i a}$ and $\boldsymbol{\psi}_{a \alpha}$. Similarly to the discussion in section 3 , the Goldstone fermions $\psi_{a \alpha}$ may enter the component action only through the einbein $\mathcal{E}$ and the covariant derivatives $\mathcal{D}_{t} q_{i a}$, defined now as

$$
\partial_{t}=\mathcal{E} \mathcal{D}_{t}, \quad \mathcal{E}=1+\frac{1}{2} \Omega^{\beta \gamma} \psi_{\beta}^{a} \partial_{t} \psi_{a \gamma}, \quad \mathcal{E}^{-1}=1-\frac{1}{2} \Omega^{\beta \gamma} \psi_{\beta}^{a} \mathcal{D}_{t} \psi_{a \gamma},
$$

Keeping in the mind the known bosonic limit of the action (4.17), we come to the unique candidate of the component on-shell action

$$
S=\alpha \int d t+(1-\alpha) \int d t \mathcal{E}-\int d t \mathcal{E} \sqrt{1-2 \mathcal{D}_{t} q^{i a} \mathcal{D}_{t} q_{i a}} .
$$

This action is perfectly invariant with respect to broken $S$ supersymmetry, realized on the physical components and their derivatives as

$$
\delta_{S}^{*} q_{i a}=\frac{1}{2} \eta_{\alpha}^{b} \psi_{b \beta} \Omega^{\alpha \beta} \partial_{t} q_{i a}, \quad \delta_{S}^{*}\left(\mathcal{D}_{t} q_{i a}\right)=\frac{1}{2} \eta_{\alpha}^{b} \psi_{b \beta} \Omega^{\alpha \beta} \partial_{t}\left(\mathcal{D}_{t} q_{i a}\right), \quad \delta_{S}^{*} \psi_{a \alpha}=\eta_{a \alpha}+\frac{1}{2} \eta_{\beta}^{b} \psi_{b \lambda} \Omega^{\beta \lambda} \partial_{t} \psi_{a \alpha} .
$$

From (4.21) one may find the transformation properties of the einbein $\mathcal{E}$

$$
\delta_{S}^{*} \mathcal{E}=\frac{1}{2} \eta_{\alpha}^{a} \partial_{t}\left(\mathcal{E} \Omega^{\alpha \beta} \psi_{a \beta}\right)
$$

Now, combining (4.21) and (4.22), we will get

$$
\delta_{S}^{*}\left(\mathcal{E F}\left[\mathcal{D}_{t} q^{j b} \mathcal{D}_{t} q_{j b}\right]\right)=\frac{1}{2} \eta_{\alpha}^{a} \partial_{t}\left(\Omega^{\alpha \beta} \psi_{a \beta} \mathcal{E} \mathcal{F}\left[\mathcal{D}_{t} q^{j b} \mathcal{D}_{t} q_{j b}\right]\right)
$$


and, therefore, the second and the third terms in the action (4.20) are separately invariant with respect to $S$ supersymmetry. The first term in (4.20) is trivially invariant with respect to both, broken and unbroken supersymmetries.

The last step is to impose invariance with respect to unbroken $Q$ supersymmetry. Under the transformations of unbroken supersymmetry taken in the fixed point the variation of any superfield reads

$$
\delta_{Q}^{*} \boldsymbol{F}=-\varepsilon_{i}^{\alpha} Q_{\alpha}^{i} \boldsymbol{F}
$$

From this one may find the variations of the components $q_{i a}$ and $\psi_{a \alpha}$ and their covariant derivatives:

$$
\begin{aligned}
& \delta_{Q}^{*} q_{i a}=-\varepsilon_{i}^{\alpha} \psi_{a \alpha}+\frac{1}{4} \varepsilon_{j}^{\alpha} \lambda^{j b} \psi_{b \alpha} \partial_{t} q_{i a}, \\
& \delta_{Q}^{*}\left(\mathcal{D}_{t} q_{i a}\right)=-\varepsilon_{i}^{\alpha} \mathcal{D}_{t} \psi_{a \alpha}+\frac{1}{4} \varepsilon_{j}^{\alpha} \frac{\lambda_{i a}}{1+\frac{1}{8} \lambda^{2}} \lambda^{j b} \mathcal{D}_{t} \psi_{b \alpha}+\frac{1}{4} \varepsilon_{j}^{\alpha} \lambda^{j b} \psi_{b \alpha} \partial_{t}\left(\mathcal{D}_{t} q_{i a}\right), \\
& \delta_{Q}^{*} \psi_{a \alpha}=\frac{1}{2} \varepsilon_{j}^{\beta} \Omega_{\alpha \beta} \lambda_{a}^{j}+\frac{1}{4} \varepsilon_{j}^{\beta} \lambda^{j b} \psi_{b \beta} \partial_{t} \psi_{a \alpha} .
\end{aligned}
$$

The variation of the einbein $\mathcal{E}$ can be also computed and it reads

$$
\delta_{Q}^{*} \mathcal{E}=\frac{1}{4} \varepsilon_{j}^{\beta} \partial_{t}\left(\mathcal{E} \lambda^{j b} \psi_{b \beta}\right)-\frac{1}{2} \varepsilon_{j}^{\beta} \lambda^{j b} \partial_{t} \psi_{b \beta} .
$$

It is a matter of lengthy, but straightforward calculations to check that the action (4.20) is invariant under unbroken supersymmetry (4.24), (4.25) if $\alpha=2$.

Thus, the component action, invariant under both unbroken and broken $N=8$ supersymmetries, reads

$$
S=\int d t\left[2-\mathcal{E}\left(1+\sqrt{1-2 \mathcal{D}_{t} q^{i a} \mathcal{D}_{t} q_{i a}}\right)\right] .
$$

\section{Supermembrane in $\mathrm{D}=4$}

As an instructive application of our approach we consider in this section as an example two models, namely, the supermembrane in $D=4$ and the supersymmetric space-filling D2-brane. We will mainly follow the paper [9].

\subsection{Supermembrane in $\mathrm{D}=4$ : kinematical constraints, equations of motion and the component action}

The nonlinear realization of the breaking $N=1, D=4 \rightarrow N=1, d=3$ has been constructed in 9]. There, the $N=1, D=4$ super Poincaré group has been realized in its coset over the $d=3$ Lorentz group $S O(1,2)$

$$
g=e^{x^{a b} P_{a b}} e^{\theta^{a} Q_{a}} e^{\boldsymbol{q} Z} e^{\boldsymbol{\psi}^{a} S_{a}} e^{\boldsymbol{\Lambda}^{a b} K_{a b}}
$$

Here, $x^{a b}, \theta^{a}$ are $N=1, d=3$ superspace coordinates, while the remaining coset parameters are Goldstone superfields, $\boldsymbol{\psi}^{a}=\boldsymbol{\psi}^{a}(x, \theta), \boldsymbol{q}=\boldsymbol{q}(x, \theta), \boldsymbol{\Lambda}^{a b}=\boldsymbol{\Lambda}^{a b}(x, \theta)$. To reduce the number of independent superfields one has to impose the constraints

$$
\Omega_{Z}=0 \Rightarrow\left\{\begin{array}{l}
\nabla_{a b} \boldsymbol{q}+\frac{4}{1+2 \boldsymbol{\lambda}^{2}} \boldsymbol{\lambda}_{a b}=0, \\
\nabla_{a} \boldsymbol{q}-\boldsymbol{\psi}_{a}=0 .
\end{array}\right.
$$

Eqs. (5.2) allow us to express $\boldsymbol{\lambda}_{a b}(x, \theta)$ and $\boldsymbol{\psi}^{a}(x, \theta)$ through covariant derivatives of $\boldsymbol{q}(x, \theta)$. Thus, the bosonic superfield $\boldsymbol{q}(x, \theta)$ is the only essential Goldstone superfield we need for this case of the partial breaking of the global supersymmetry. The constraints (5.2) are covariant under all symmetries and they do not imply any dynamics and leave $\boldsymbol{q}(x, \theta)$ off-shell.

\footnotetext{
${ }^{5}$ We collect the exact expressions for the covariant derivatives $\nabla_{a b}, \nabla_{a}$ and their properties, constructed in [9], in Appendix A.
} 
The last step we can make within the coset approach is to write the covariant superfield equations of motion. It was shown in 9 , that this can be achieved by imposing the following constraint on the Cartan form:

$$
\Omega_{S} \mid=0 \Rightarrow\left\{\begin{array}{l}
\nabla^{a} \boldsymbol{\psi}_{a}=0 \\
\nabla_{(a} \boldsymbol{\psi}_{b)}=-2 \boldsymbol{\lambda}_{a b}
\end{array}\right.
$$

where | denotes the ordinary $d \theta$-projection of the form $\Omega_{S}$.

Eqs. (5.3) imply the proper dynamical equation of motion

$$
\nabla^{a} \nabla_{a} \boldsymbol{q}=0 .
$$

This equation is also covariant with respect to all symmetries, and its bosonic limit for $q(x)=\left.\boldsymbol{q}(x, \theta)\right|_{\theta=0}$ reads

$$
\partial_{a b}\left(\frac{\partial^{a b} q}{\sqrt{1-\frac{1}{2} \partial q \cdot \partial q}}\right)=0,
$$

which corresponds to the "static gauge" form of the $D=4$ membrane Nambu-Goto action

$$
S=\int d^{3} x\left(1-\sqrt{1-\frac{1}{2} \partial^{a b} q \partial_{a b} q}\right) .
$$

Thus, the equations (5.3) indeed describe the supermembrane in $D=4$.

Until now we just repeated the standard coset approach steps from the paper [9] in the application to the $N=1, D=4$ supermembrane. As was already mentioned in section 2 , the nonlinear realization approach fails to construct the superfield action. That is why, to construct the superfield action one has to involve some additional arguments/scheme as it has been done, for example, in [9].

Funny enough, if we instead will be interested in the component action, then it can be constructed almost immediately within the nonlinear realization approach. One may check that all important features of the on-shell (i.e. with Eqs. (5.3) taken into account) component action we summarized in section 2, are present in the case at hands. Indeed,

- All physical components, i.e. $\left.\boldsymbol{q}\right|_{\theta=0}$ and $\left.\boldsymbol{\psi}^{a}\right|_{\theta=0}$, are among the "coordinates" of our coset (5.1) as the $\theta=0$ parts of the corresponding superfields,

- Under spontaneously broken supersymmetry the superspace coordinates $\theta^{a}$ do not transform at all (A.5). Therefore, the corresponding transformation properties of the fermionic components $\left.\boldsymbol{\psi}^{a}\right|_{\theta=0}$ are the same as in the Volkov-Akulov model [3], where all supersymmetries are supposed to be spontaneously broken,

- Finally, the $\theta=0$ component of our essential Goldstone superfield $\boldsymbol{q}(x, \theta)$ does not transform under spontaneously broken supersymmetry and, therefore, it behaves like a "matter" field within the Volkov-Akulov scheme.

As the immediate consequences of these features we conclude that

- The fermionic components $\left.\boldsymbol{\psi}^{a}\right|_{\theta=0}$ may enter the component action either through $\operatorname{det} \mathcal{E}$ (A.14) (to compensate the transformation of volume $d^{3} x$ under (A.5)) or through the covariant derivatives $\mathcal{D}_{a b}$ (A.12), only,

- The "matter" field $q=\left.\boldsymbol{q}\right|_{\theta=0}$ may enter the action only through covariant derivatives $\mathcal{D}_{a b} q$.

Thus, the unique candidate to be the component on-shell action, invariant with respect to spontaneously broken supersymmetry $S$ reads

$$
S=\alpha \int d^{3} x+\beta \int d^{3} x \operatorname{det} \mathcal{E} \mathcal{F}\left(\mathcal{D}^{a b} q \mathcal{D}_{a b} q\right),
$$

with an arbitrary, for the time being, function $\mathcal{F}$. All other interactions between the bosonic component $q$ and the fermions of spontaneously broken supersymmetry $\psi^{a}$ are forbidden! 
Note, that the first, trivial term in (5.7) is independently invariant under broken (and unbroken!) supersymmetries, because, in virtue of (A.5)

$$
\delta_{S} \int d^{3} x \sim \int d^{3} x \partial_{a b}\left(\xi^{a} \psi^{b}\right) \quad \text { and, therefore } \quad \delta_{S} \int d^{3} x=0 .
$$

As we already said in section 2, this term in the action (5.7) ensures the validity of the limit $S_{q=0, \psi=0}=0$.

The action (5.7) is the most general component action invariant with respect to broken supersymmetry. But in the present case we explicitly know its bosonic limit - it should be just the Nambu-Goto action (5.6). Some additional information about its structure comes from the linearized form of the action, which, according with its invariance with respect to unbroken supersymmetry, has to be

$$
S_{\text {lin }} \sim \psi^{a} \partial_{a b} \psi^{b}-\frac{1}{4} \partial^{a b} q \partial_{a b} q .
$$

Combining all these ingredients, which completely fix the parameters $\alpha$ and $\beta$ in (5.7), we can write the component action of $N=1, D=4$ supermembrane as

$$
S=\int d^{3} x\left[2-\operatorname{det} \mathcal{E}\left(1+\sqrt{1-\frac{1}{2} \mathcal{D}^{a b} q \mathcal{D}_{a b} q}\right)\right] .
$$

The explicit expression for $\operatorname{det} \mathcal{E}$ has the form

$$
\begin{aligned}
\operatorname{det} \mathcal{E} & =1+\frac{1}{2} \psi^{a} \mathcal{D}_{a b} \psi^{b}-\frac{1}{16} \psi^{d} \psi_{d} \mathcal{D}^{a b} \psi^{c} \mathcal{D}_{a b} \psi_{c}= \\
& =1+\frac{1}{2} \psi^{a} \partial_{a b} \psi^{b}+\frac{1}{8} \psi^{d} \psi_{d}\left(\partial^{a b} \psi_{b} \partial_{a c} \psi^{c}+\frac{1}{2} \partial^{a b} \psi^{c} \partial_{a b} \psi_{c}\right) .
\end{aligned}
$$

Let us stress, that such a simple form of the component action is achieved only in the rather specific basis, where the bosonic $q$ and fermionic fields $\psi^{a}$ are the Goldstone fields of the nonlinear realization. Surely, this choice is not unique and in different bases the explicit form of action could drastically change. The most illustrative example is given by the action in [24, where the on-shell component action for the supermembrane has been constructed for the first time.

The detailed proof that the action (5.10) is invariant with respect to both, broken and unbroken supersymmetries, can be found in Appendix B.

\subsection{Supersymmetric space-filling D2-brane}

Due to the duality between scalar field and gauge field strength in $d=3$, the action for D2-brane can be easily constructed within the coset approach. The idea of the construction is similar to the purely bosonic case. The crucial step is to treat the first, bosonic component of $\boldsymbol{\lambda}_{a b}$ as an independent component (i.e. to ignore the (a) part of Eqs.(5.2) ). Now, the generalized variant of the action (5.10) reads

$$
S=\int d^{3} x\left[2-\operatorname{det} \mathcal{E}-\operatorname{det} \mathcal{E}\left(1+2 \frac{\lambda^{a b}\left(\mathcal{D}_{a b} q+2 \lambda_{a b}\right)}{1-2 \lambda^{2}}\right)\right] .
$$

All these summands have a description in terms of $\theta=0$ parts of the Cartan forms (A.9). The first term is just a volume form constructed from ordinary differentials $d x^{a b}$. The second terms is a volume form constructed from semi-covariant differentials $d \hat{x}^{a b}$

$$
d \hat{x}^{a b}=d x^{a b}+\frac{1}{4} \psi^{a} d \psi^{b}+\frac{1}{4} \psi^{b} d \psi^{a} .
$$

Finally, the last term in (5.12) is a volume form constructed from the $\theta=0$ component of the form $\Omega_{P}^{a b}$ (A.9)

$$
d \tilde{x}^{a b}=d \hat{x}^{a b}+\frac{2}{1-2 \lambda^{2}} \lambda^{a b}\left(\mathcal{D}_{c d} q+2 \lambda_{c d}\right) d \hat{x}^{c d} .
$$


Since the action (5.12) depends only on $\lambda^{a b}$ and not on its derivatives, the $\lambda$-equation of motion

$$
\mathcal{D}_{a b} q=-\frac{4 \lambda_{a b}}{1+2 \lambda^{2}}
$$

can be used to eliminate $\lambda^{a b}$ in favor of $\mathcal{D}_{a b} q$. Clearly, the Eq. (5.13) is just the (a) part of the constraints (5.2), we ignored while introducing the action (5.12). Plugging $\lambda_{a b}$ expressed through $\mathcal{D}_{a b} q$ back into (5.12) gives us the action (5.10).

Alternatively, the equation of motion for $q$

$$
\partial_{a b}\left[\frac{\operatorname{det} \mathcal{E} \lambda^{c d}\left(\mathcal{E}^{-1}\right)_{c d}^{a b}}{1-2 \lambda^{2}}\right]=0
$$

has the form of the $d=3$ Bianchi identity for the field strength $F^{a b}$

$$
F^{a b} \equiv \frac{\operatorname{det} \mathcal{E} \lambda^{c d}\left(\mathcal{E}^{-1}\right)_{c d}^{a b}}{1-2 \lambda^{2}} \quad \Rightarrow \quad \partial_{a b} F^{a b}=0 .
$$

Substituting this into the action (5.12) and integrating by parts, one may bring it to the supersymmetric D2-brane action

$$
S=\int d^{3} x\left[2-\operatorname{det} \mathcal{E}\left(1+\sqrt{1+8 \widetilde{F}^{2}}\right)\right]
$$

where

$$
\widetilde{F}_{a b} \equiv \frac{\mathcal{E}_{a b}{ }^{c d} F_{c d}}{\operatorname{det} \mathcal{E}}=\frac{\lambda_{a b}}{1-2 \lambda^{2}}
$$

Therefore,

$$
S=2 \int d^{3} x\left[1-\operatorname{det} \mathcal{E} \frac{1}{1-2 \lambda^{2}}\right]
$$

Clearly, in the bosonic limit $\widetilde{F}_{a b}=F_{a b}$ and thus, the bosonic part of the (5.16) is the standard Born-Infeld action for D2-brane, as it should be.

\section{Supermembrane in $\mathrm{D}=5$}

In this section we construct the on-shell component action for $N=1, D=5$ supermembrane and its dual versions, corresponding to a vector and a double vector supermultiplets. We demonstrate that the proper choice of the components and using the covariant (with respect to broken supersymmetry) derivatives drastically simplify the action: it can be represented as the sum of four terms each having an explicit geometric meaning.

\subsection{Supermembrane}

In the present case we are dealing with spontaneous breaking of $N=1, D=5$ Poincaré supersymmetry down to $N=2, d=3$ one. From the $d=3$ standpoint the $N=1, D=5$ supersymmetry algebra is a central-charges extended $N=4$ Poincaré superalgebra with the following basic anticommutation relations:

$$
\left\{Q_{a}, \bar{Q}_{b}\right\}=2 P_{a b}, \quad\left\{S_{a}, \bar{S}_{b}\right\}=2 P_{a b}, \quad\left\{Q_{a}, S_{b}\right\}=2 \epsilon_{a b} Z, \quad\left\{\bar{Q}_{a}, \bar{S}_{b}\right\}=2 \epsilon_{a b} \bar{Z} .
$$

The $d=3$ translations generator $P_{a b}$ and the central charge generators $Z, \bar{Z}$ form $D=5$ translation generators. We will also split the generators of $D=5$ Lorentz algebra $s o(1,4)$ into $d=3$ Lorentz algebra generators $M_{a b}$, the generators $K_{a b}$ and $\bar{K}_{a b}$ belonging to the coset $S O(1,4) / S O(1,2) \times U(1)$ and $U(1)$ generator $J$. The full set of (anti)commutation relations can be found in the Appendix C. 
Keeping $d=3$ Lorentz and, commuting with it, $U(1)$ subgroups of $D=5$ Lorentz group $S O(1,4)$ linearly realized, we will choose the coset element as

$$
g=e^{i x^{a b} P_{a b}} e^{\theta^{a} Q_{a}+\bar{\theta}^{a} \bar{Q}_{a}} e^{i(\boldsymbol{q} Z+\overline{\boldsymbol{q}} \bar{Z})} e^{\boldsymbol{\psi}^{a} S_{a}+\overline{\boldsymbol{\psi}}^{a} \bar{S}_{a}} e^{i\left(\boldsymbol{\Lambda}^{a b} K_{a b}+\overline{\boldsymbol{\Lambda}}^{a b} \bar{K}_{a b}\right)} .
$$

Here, $\left\{x^{a b}, \theta^{a}, \bar{\theta}^{a}\right\}$ are $N=2, d=3$ superspace coordinates, while the remaining coset parameters are $N=2$ Goldstone superfields. The whole $N=1, D=5$ Poincaré supergroup can be realized in this coset by the left acting on (6.2) of the different elements of the supergroup. We summarize in Appendix $\mathrm{C}$ the resulting transformation properties of the coordinates and superfields with respect to unbroken (C.6), broken (C.7) supersymmetries and automorphism (C.8), as well as a pure technical calculation of Cartan forms, semi-covariant derivatives and their superalgebra (C.11), (C.14), (C.18).

Similarly to the previously considered cases, to reduce the number of independent superfields one has to impose the constraints

$$
\Omega_{Z}=0 \Rightarrow\left\{\begin{array}{l}
\nabla_{a b} \boldsymbol{q}=-2 i \frac{(1+\boldsymbol{l} \cdot \overline{\boldsymbol{l}}) \boldsymbol{l}_{a b}-\boldsymbol{l}^{2} \overline{\boldsymbol{l}}_{a b}}{(1+\boldsymbol{l} \cdot \bar{\cdot})^{2}-\boldsymbol{l}^{2} \bar{l}^{2}}, \\
\nabla_{a} \boldsymbol{q}=-2 i \boldsymbol{\psi}_{a}, \quad \nabla_{a} \boldsymbol{q}=0,
\end{array} \bar{\Omega}_{Z}=0 \quad \Rightarrow \quad\left\{\begin{array}{l}
\nabla_{a b} \overline{\boldsymbol{q}}=2 i \frac{(1+\boldsymbol{l} \cdot \bar{l}) \overline{\boldsymbol{l}}_{a b}-\overline{\boldsymbol{l}}^{2} \boldsymbol{l}_{a b}}{\left(1+\boldsymbol{l} \cdot \overline{l^{2}}-\boldsymbol{l}^{2} \overline{\bar{l}}^{2}\right.}, \\
\bar{\nabla}_{a} \overline{\boldsymbol{q}}=-2 i \overline{\boldsymbol{\psi}}_{a}, \quad \nabla_{a} \overline{\boldsymbol{q}}=0 .
\end{array}\right.\right.
$$

Here, in order to simplify the expressions, we have passed to the some variant of the stereographic parametrization of the coset $S O(1,4) / S O(1,2) \times U(1)$

$$
\boldsymbol{l}_{a b}=\left(\frac{\tanh \sqrt{\boldsymbol{Y}}}{\sqrt{\boldsymbol{Y}}}\right)_{a b}^{c d} \boldsymbol{\Lambda}_{c d}, \quad \overline{\boldsymbol{l}}_{a b}=\left(\frac{\tanh \sqrt{\boldsymbol{Y}}}{\sqrt{\boldsymbol{Y}}}\right)_{a b}^{c d} \overline{\boldsymbol{\Lambda}}_{c d}
$$

The equations (6.3) allow us to express superfields $\boldsymbol{\Lambda}_{a b}, \overline{\boldsymbol{\Lambda}}_{a b}$ and $\boldsymbol{\psi}^{a}, \overline{\boldsymbol{\psi}}^{a}$ through covariant derivatives of $\boldsymbol{q}(x, \theta, \bar{\theta})$ and $\overline{\boldsymbol{q}}(x, \theta, \bar{\theta})$. Thus, the bosonic superfields $\boldsymbol{q}(x, \theta, \bar{\theta}), \overline{\boldsymbol{q}}(x, \theta, \bar{\theta})$ are the only essential Goldstone superfields needed for this case of the partial breaking of the global supersymmetry. The constraints (6.3) are covariant under all symmetries, they do not imply any dynamics and leave $\boldsymbol{q}(x, \theta, \bar{\theta})$ and $\overline{\boldsymbol{q}}(x, \theta, \bar{\theta})$ off-shell.

Within the coset approach we may also write the covariant superfield equations of motion. This can be achieved by imposing the proper constraint on the Cartan forms for broken supersymmetry. In the present case these constraints read

$$
\begin{aligned}
& \Omega_{S} \mid=0 \Rightarrow(a) \nabla_{a} \boldsymbol{\psi}_{b}=0, \quad(b) \bar{\nabla}_{b} \psi^{a}=-i \boldsymbol{\Lambda}_{b}{ }^{c}\left(\frac{\tan 2 \sqrt{\overline{\boldsymbol{T}}}}{\sqrt{\overline{\boldsymbol{T}}}}\right)_{c}^{a} \equiv-i \boldsymbol{\lambda}_{b}^{a} \\
& \bar{\Omega}_{S} \mid=0 \Rightarrow(a) \bar{\nabla}_{a} \overline{\boldsymbol{\psi}}_{b}=0, \quad(b) \nabla_{b} \overline{\boldsymbol{\psi}}^{a}=i \boldsymbol{\Lambda}_{b}{ }^{c}\left(\frac{\tan 2 \sqrt{\boldsymbol{T}}}{\sqrt{\boldsymbol{T}}}\right)_{c}^{a} \equiv i, \overline{\boldsymbol{\lambda}}_{b}^{a},
\end{aligned}
$$

where $\mid$ means the $d \theta$-projection of the forms.

Let us make a few comments concerning the constraints given above:

- The easiest way to check that the equations (6.3), (6.5) put the theory on-shell is to consider these equations in the linearized form

$$
\begin{aligned}
& \partial_{a b} \boldsymbol{q}=-2 i \boldsymbol{\Lambda}_{a b}(a), \quad D_{a} \boldsymbol{q}=-2 i \boldsymbol{\psi}_{a}(b), \quad \bar{D}_{a} \boldsymbol{q}=0(c), \\
& D_{a} \boldsymbol{\psi}_{b}=0(a), \quad \bar{D}_{b} \boldsymbol{\psi}^{a}=-2 i \boldsymbol{\Lambda}_{b}^{a}(b) .
\end{aligned}
$$

Acting on eq. (6.6b) by $\bar{D}_{b}$ and using the eq. (6.6 c) and the algebra of spinor derivatives (C.15) we immediately conclude that eq. (6.7 b) follows from (6.6). In addition, the eq. (6.7 $\mathrm{b})$ means that the auxiliary component of the superfield $\boldsymbol{q}$ is zero and, therefore, our system is on-shell

$$
D_{a} \boldsymbol{\psi}_{b}=0 \Rightarrow D^{2} \boldsymbol{q}=0 \Rightarrow \partial_{a b} D^{b} \boldsymbol{q}=0 \Rightarrow \square \boldsymbol{q}=0 .
$$


- It turns out that the variables $\left\{\boldsymbol{\lambda}_{a}^{b}, \overline{\boldsymbol{\lambda}}_{a}^{b}\right\}$ defined in (6.5), are more suitable than the $\left\{\boldsymbol{l}_{a b}, \overline{\boldsymbol{l}}_{a b}\right\}$ (6.4) one. Using the algebra of covariant derivatives (‥18) it is easy to find the following relations from (6.3) and (6.5):

$$
\nabla_{a b} \boldsymbol{q}=-i \frac{\boldsymbol{\lambda}_{a b}-\frac{1}{2} \boldsymbol{\lambda}^{2} \overline{\boldsymbol{\lambda}}_{a b}}{1-\frac{1}{4} \boldsymbol{\lambda}^{2} \overline{\boldsymbol{\lambda}}^{2}}, \quad \nabla_{a b} \overline{\boldsymbol{q}}=i \frac{\overline{\boldsymbol{\lambda}}_{a b}-\frac{1}{2} \overline{\boldsymbol{\lambda}}^{2} \boldsymbol{\lambda}_{a b}}{1-\frac{1}{4} \boldsymbol{\lambda}^{2} \overline{\boldsymbol{\lambda}}^{2}} .
$$

These equations play the same role as those in (6.3), relating the superfields $\left\{\boldsymbol{\lambda}_{a b}, \overline{\boldsymbol{\lambda}}_{a b}\right\}$ (and, therefore, the superfields $\left.\left\{\boldsymbol{\Lambda}_{a b}, \overline{\boldsymbol{\Lambda}}_{a b}\right\}\right)$ with the space-time derivatives of the superfields $\{\boldsymbol{q}, \overline{\boldsymbol{q}}\}$.

Now we present two different ways to construct the bosonic action.

The first of them is based on the consideration of the bosonic coset related to (6.2) and on the invariance of constraints (6.3), (6.5) with respect to all $N=1, D=5$ Poincaré supergroup. Thus we have

$$
g_{b o s}=e^{i x^{a b} P_{a b}} e^{i(q Z+\bar{q} \bar{Z})} e^{i\left(\Lambda^{a b} K_{a b}+\bar{\Lambda}^{a b} \bar{K}_{a b}\right)} .
$$

Clearly, the corresponding bosonic Cartan forms can be easily extracted from their superfields version (C.10). The bosonic version of the constraints (6.3) results in the relations

$$
\partial_{a b} q=-2 i \frac{(1+l \cdot \bar{l}) l_{a b}-l^{2} \bar{l}_{a b}}{(1+l \cdot \bar{l})^{2}-l^{2} \bar{l}^{2}}, \quad \partial_{a b} \bar{q}=2 i \frac{(1+l \cdot \bar{l}) \bar{l}_{a b}-\bar{l}^{2} l_{a b}}{(1+l \cdot \bar{l})^{2}-l^{2} \bar{l}^{2}}
$$

while the bosonic vielbein $\mathcal{B}_{a b}{ }^{c d}=\left.\mathcal{E}_{a b}{ }^{c d}\right|_{\psi=0}$

$$
\left(\Omega_{P}^{b o s}\right)=d x^{a b} \mathcal{B}_{a b}{ }^{c d} P_{c d}
$$

acquires the form

$$
\mathcal{B}_{a b}^{c d}=\delta_{a}^{(c} \delta_{b}^{d)}-\frac{2}{(1+l \cdot \bar{l})^{2}-l^{2} \bar{l}^{2}}\left[(1+l \cdot \bar{l})\left(\bar{l}^{c d} l_{a b}+l^{c d} \bar{l}_{a b}\right)-\bar{l}^{2} l^{c d} l_{a b}-l^{2} \bar{l}^{c d} \bar{l}_{a b}\right] .
$$

Therefore, the simplest invariant bosonic action reads

$$
S_{\text {bos }}=\int d^{3} x \operatorname{det} \mathcal{B}=\int d^{3} x \frac{(1-l \cdot \bar{l})^{2}-l^{2} \bar{l}^{2}}{(1+l \cdot \bar{l})^{2}-l^{2} \bar{l}^{2}},
$$

or in terms of $\{q, \bar{q}\}$

$$
S_{b o s}=\int d^{3} x \sqrt{\left(1-\partial_{a b} q \partial^{a b} \bar{q}\right)^{2}-\left(\partial_{a b} q \partial^{a b} q\right)\left(\partial_{c d} \bar{q} \partial^{c d} \bar{q}\right)} .
$$

The latter is the static gauge Nambu-Goto action for membrane in $D=5$. One can also add the following action, trivially invariant under the transformations $I S O(1,4)$

$$
S_{0}=\int d^{3} x
$$

Another way to derive the bosonic action is to use automorphism transformation laws. These laws (C.8) in the bosonic limit have the form

$$
\delta x^{a b}=2 i\left(\bar{a}^{a b} q-a^{a b} \bar{q}\right), \quad \delta q=-2 i(a x), \quad \delta \bar{q}=2 i(\bar{a} x) .
$$

The active form of these transformations reads

$$
\delta^{*} q=-2 i(a x)-2 i \partial_{a b} q\left(\bar{a}^{a b} q-a^{a b} \bar{q}\right), \quad \delta^{*} \bar{q}=2 i(\bar{a} x)-2 i \partial_{a b} \bar{q}\left(\bar{a}^{a b} q-a^{a b} \bar{q}\right) .
$$

Due to translations, $U(1)$-rotations and $d=3$ Lorentz invariance, the action may depend only on the following scalar combination of partial derivatives of bosons $\{q, \bar{q}\}$

$$
\xi=\partial_{a b} q \partial^{a b} \bar{q}, \quad \eta=\partial_{a b} q \partial^{a b} q, \quad \bar{\eta}=\partial_{a b} \bar{q} \partial^{a b} \bar{q},
$$


which in accordance with (6.17) transforms as

$$
\begin{aligned}
\delta^{*} \xi= & 2 i(\bar{a} \partial q)-2 i(a \partial \bar{q})-2 i\left(\bar{a}^{a b} q-a^{a b} \bar{q}\right) \partial_{a b} \xi-2 i(\bar{a} \partial q) \xi+2 i(a \partial \bar{q}) \xi-2 i(\bar{a} \partial \bar{q}) \eta+2 i(a \partial q) \bar{\eta} \\
\delta^{*}(\eta \bar{\eta})= & 4 i(\bar{a} \partial \bar{q}) \eta-4 i(a \partial q) \bar{\eta}-2 i\left(\bar{a}^{k l} q-a^{k l} \bar{q}\right) \partial_{k l}(\eta \bar{\eta})-4 i(\bar{a} \partial q) \eta \bar{\eta}+4 i(a \partial \bar{q}) \eta \bar{\eta}+ \\
& 4 i(a \partial q) \xi \bar{\eta}-4 i(\bar{a} \partial \bar{q}) \xi \eta
\end{aligned}
$$

Therefore, the variation of the arbitrary function $\mathcal{F}(\xi, \eta \bar{\eta})$ reads

$$
\begin{aligned}
\delta^{*} \mathcal{F}= & 2 i[(a \partial q) \bar{\eta}-(\bar{a} \partial \bar{q}) \eta]\left(\mathcal{F}_{\xi}+2(\xi-1) \mathcal{F}_{(\eta \bar{\eta})}\right)+2 i[(\bar{a} \partial q)-(a \partial \bar{q})]\left(\mathcal{F}+(1-\xi) \mathcal{F}_{\xi}-2 \eta \bar{\eta} \mathcal{F}_{(\eta \bar{\eta})}\right) \\
& -2 i \partial_{a b}\left[\left(q \bar{a}^{a b}-\bar{q} a^{a b}\right) \mathcal{F}\right] .
\end{aligned}
$$

Thus, to achieve the invariance of the action one has impose the following restrictions on the function $\mathcal{F}$ :

$$
\mathcal{F}_{\xi}+2(\xi-1) \mathcal{F}_{(\eta \bar{\eta})}=0, \quad \mathcal{F}+\mathcal{F}_{\xi}(1-\xi)-2(\eta \bar{\eta}) \mathcal{F}_{(\eta \bar{\eta})}=0
$$

with the evident solution

$$
\mathcal{F}=\sqrt{(1-\xi)^{2}-\eta \bar{\eta}}
$$

Therefore, the invariant action has the form

$$
S=\int d^{3} x \sqrt{\left(1-\partial_{a b} q \partial^{a b} \bar{q}\right)^{2}-\left(\partial_{a b} q \partial^{a b} q\right)\left(\partial_{k l} \bar{q} \partial^{k l} \bar{q}\right)},
$$

and thus, it coincides with that previously constructed in (6.14), as it should be.

Let us now construct the full component action for supermembrane which will be invariant under both broken and unbroken supersymmetries. We begin our analysis with the broken supersymmetry $S$.

The superspace coordinates $\{\theta, \bar{\theta}\}$ of the coset (6.2) do not transform under $S$ supersymmetry. Therefore, each component of superfields transforms independently under the broken supersymmetry. Thus, from (C.7) one finds

$$
\delta x^{a b}=i\left(\varepsilon^{(a} \bar{\psi}^{b)}+\bar{\varepsilon}^{(a} \psi^{b)}\right), \quad \delta q=0, \quad \delta \bar{q}=0, \quad \delta \psi^{a}=\varepsilon^{a}, \quad \delta \bar{\psi}^{a}=\bar{\varepsilon}^{a} .
$$

Then, one may easily check that the $\theta=0$ projections of the covariant differential $\triangle x^{a b}$ (C.10)

$$
\left.\hat{\triangle} x^{a b} \equiv \triangle x^{a b}\right|_{\theta=0}=d x^{a b}-i\left(\psi^{(a} d \bar{\psi}^{b)}+\bar{\psi}^{(a} d \psi^{b)}\right) \equiv \mathcal{E}_{c d}^{a b} d x^{c d},
$$

as well as the covariant derivatives constructed from them

$$
\mathcal{D}_{a b}=\left(\mathcal{E}^{-1}\right)_{a b}^{c d} \partial_{c d}
$$

are also invariant under broken supersymmetry. From all this it immediately follows that the action possessing the proper bosonic limit (6.14) and invariant under broken supersymmetry reads

$$
S_{1}=\int d^{3} x \operatorname{det} \mathcal{E} \sqrt{\left(1-\mathcal{D}_{a b} q \mathcal{D}^{a b} \bar{q}\right)^{2}-\left(\mathcal{D}_{a b} q \mathcal{D}^{a b} q\right)\left(\mathcal{D}_{c d} \bar{q} \mathcal{D}^{c d} \bar{q}\right)} .
$$

The action (6.26) reproduces the kinetic terms for the bosonic and fermionic components

$$
S_{1}=\int d^{3} x\left[-i\left(\psi^{a} \partial_{a b} \bar{\psi}^{b}+\bar{\psi}^{a} \partial_{a b} \psi^{b}\right)-\partial_{a b} q \partial^{a b} \bar{q}+\ldots\right],
$$

but the coefficient between them is strictly fixed. This could be not enough to maintain unbroken supersymmetry. So, one has to add to (6.26) the purely fermionic action

$$
S_{2}=\int d^{3} x \operatorname{det} \mathcal{E}
$$


which is trivially invariant under broken supersymmetry. Finally, in order to have a proper limit

$$
S_{q \rightarrow 0, \psi \rightarrow 0}=0,
$$

one has to involve into the game the trivial action $S_{0}$ that reads as

$$
S_{0}=\int d^{3} x
$$

Thus, the Ansatz for the supersymmetric action acquires the form

$$
\begin{aligned}
S & =(1+\alpha) S_{0}-S_{1}-\alpha S_{2} \\
& =(1+\alpha) \int d^{3} x-\int d^{3} x \operatorname{det} \mathcal{E}\left(\alpha+\sqrt{\left(1-\mathcal{D}_{a b} q \mathcal{D}^{a b} \bar{q}\right)^{2}-\left(\mathcal{D}_{a b} q \mathcal{D}^{a b} q\right)\left(\mathcal{D}_{c d} \bar{q} \mathcal{D}^{c d} \bar{q}\right)}\right)
\end{aligned}
$$

where the constant $\alpha$ has to be defined.

In the previously considered cases in the above sections, the Ansatz, similar to (6.30), was completely enough to maintain the second, unbroken supersymmetry. A careful analysis shows that in the present case there is an additional Wess-Zumino term which has to be taken into account

$$
S_{W Z}=i \int d^{3} x \operatorname{det} \mathcal{E}\left(\psi^{m} \mathcal{D}_{a b} \bar{\psi}_{m}-\bar{\psi}^{m} \mathcal{D}_{a b} \psi_{m}\right) \mathcal{D}^{a c} q \mathcal{D}_{c}^{b} \bar{q}
$$

The variation of $S_{W Z}$ under $S$ supersymmetry reads (note, that only the variations of $\psi, \bar{\psi}$ without derivatives play a role)

$$
\delta S_{W Z}=i \int d^{3} x \operatorname{det} \mathcal{E}\left(\varepsilon^{m} \mathcal{D}_{a b} \bar{\psi}_{m}-\bar{\varepsilon}^{m} \mathcal{D}_{a b} \psi_{m}\right) \mathcal{D}^{a c} q \mathcal{D}_{c}{ }^{b} \bar{q}
$$

The simplest way to check that $\delta S_{W Z}=0$ is to pass to the $d=3$ vector notations 6 . Then we have

$$
\begin{aligned}
\delta S_{W Z} & \sim \int d^{3} x \operatorname{det} \mathcal{E} \epsilon^{I J K}\left(\varepsilon^{m} \mathcal{D}_{I} \bar{\psi}_{m}-\bar{\varepsilon}^{m} \mathcal{D}_{I} \psi_{m}\right) \mathcal{D}_{J} q \mathcal{D}_{K} \bar{q} \\
& \sim \int d^{3} x \operatorname{det} \mathcal{E} \operatorname{det} \mathcal{E}^{-1} \epsilon^{I J K}\left(\varepsilon^{m} \partial_{I} \bar{\psi}_{m}-\bar{\varepsilon}^{m} \partial_{I} \psi_{m}\right) \partial_{J} q \partial_{K} \bar{q} \\
& \sim \int d^{3} x \partial_{I}\left[\epsilon^{I J K}\left(\varepsilon^{m} \bar{\psi}_{m}-\bar{\varepsilon}^{m} \psi_{m}\right) \partial_{J} q \partial_{K} \bar{q}\right]=0 .
\end{aligned}
$$

Thus, the action $S_{W Z}$ (6.31) is invariant under $S$ supersymmetry and our Ansatz for the membrane action is extended to be

$$
S=(1+\alpha) S_{0}-S_{1}-\alpha S_{2}+\beta S_{W Z} .
$$

Thus, after imposing broken supersymmetry, the component action (6.34) is fixed up to two constants $\alpha$ and $\beta$. No other terms or structures are admissible!

Now we are going to demonstrate how the unbroken supersymmetry fixes these constants. In order to maintain the unbroken supersymmetry, one has to find the transformation properties of all objects presented in (6.34). Using the transformations of the superspace coordinates (C.6) one gets for the $\epsilon$-part of the transformations

$$
\begin{aligned}
& \delta \psi_{a}=-\left.\epsilon^{b}\left(D_{b} \boldsymbol{\psi}_{a}\right)\right|_{\theta=0}=\epsilon^{b} \psi^{m} \bar{\lambda}_{b}^{n} \partial_{m n} \psi_{a}, \\
& \delta \mathcal{D}_{a b} \psi_{c}=-\left.\epsilon^{d}\left(D_{d} \nabla_{a b} \boldsymbol{\psi}_{c}\right)\right|_{\theta=0}=2 \epsilon^{d} \mathcal{D}_{a b} \psi^{m} \bar{\lambda}_{d}^{n} \mathcal{D}_{m n} \psi_{b}+\epsilon^{d} \psi^{m} \bar{\lambda}_{d}^{n} \partial_{m n} \mathcal{D}_{a b} \psi_{c}, \\
& \delta \mathcal{D}_{a b} q=-\left.\epsilon^{d}\left(D_{d} \nabla_{a b} \boldsymbol{q}\right)\right|_{\theta=0}=2 \epsilon^{d} \mathcal{D}_{a b} \psi^{m} \bar{\lambda}_{d}^{n} \mathcal{D}_{m n} q+2 i \epsilon^{d} \mathcal{D}_{a b} \psi_{d}+\epsilon^{d} \psi^{m} \bar{\lambda}_{d}^{n} \partial_{m n} \mathcal{D}_{a b} q,
\end{aligned}
$$

and, as the consequence,

$$
\delta \operatorname{det} \mathcal{E}=\partial_{m n}\left[\epsilon^{d} \psi^{m} \bar{\lambda}_{d}^{n} \operatorname{det} \mathcal{E}\right]-2 \epsilon^{d} \bar{\lambda}_{d}^{n} \mathcal{D}_{m n} \psi^{n} \operatorname{det} \mathcal{E}
$$

\footnotetext{
${ }^{6}$ Our conventions to pass to/from vector indices are summarized in Appendix C, C.20.
} 
In order to fix the parameter $\alpha$ one may consider just the kinetic terms in the action (6.34)

$$
S_{k i n}=\int d^{3} x\left[-i(\alpha+1)\left(\psi^{a} \partial_{a b} \bar{\psi}^{b}+\bar{\psi}^{a} \partial_{a b} \psi^{b}\right)+\partial_{a b} q \partial^{a b} \bar{q}\right]
$$

which has to be invariant under the linearized form of the transformations (6.35) (see also (6.6), (6.7))

$$
\delta \bar{\psi}_{a}=-i \epsilon^{b} \bar{\lambda}_{b a} \simeq-\epsilon^{b} \partial_{b a} \bar{q}, \quad \delta \partial_{a b} q=2 i \epsilon^{d} \partial_{a b} \psi_{d}
$$

Varying the integrand in (6.37) and integrating by parts, we get

$$
\delta S_{k i n}=\int d^{3} x\left[2 i(\alpha+1) \epsilon^{c} \psi^{a} \partial_{a b} \partial_{c}^{b} \bar{q}-2 i \epsilon^{d} \psi_{d} \square \bar{q}\right]=\int d^{3} x\left[i(\alpha+1) \epsilon^{d} \psi_{d} \square \bar{q}-2 i \epsilon^{d} \psi_{d} \square \bar{q}\right] .
$$

Therefore, we have to fix the constant $\alpha$ as

$$
\alpha=1 \text {. }
$$

The fixing of the last parameter $\beta$ is more involved. Using the transformation properties (6.35) one may find

$$
\delta \mathcal{F}=2\left(\epsilon^{c} \bar{\lambda}_{c}^{n} \mathcal{D}_{a b} \psi^{m} \mathcal{D}_{n m} q+i \epsilon^{c} \mathcal{D}_{a b} \psi_{c}\right) \frac{\partial \mathcal{F}}{\partial \mathcal{D}_{a b} q}+2 \epsilon^{c} \bar{\lambda}_{c}^{n} \mathcal{D}_{a b} \psi^{m} \mathcal{D}_{m n} \bar{q} \frac{\partial \mathcal{F}}{\partial \mathcal{D}_{a b} \bar{q}}+\epsilon^{c} \bar{\lambda}_{c}^{n} \psi^{m} \partial_{m n} \mathcal{F}
$$

where

$$
\mathcal{F} \equiv \sqrt{\left(1-\mathcal{D}_{a b} q \mathcal{D}^{a b} \bar{q}\right)^{2}-\left(\mathcal{D}_{a b} q \mathcal{D}^{a b} q\right)\left(\mathcal{D}_{c d} \bar{q} \mathcal{D}^{c d} \bar{q}\right)}
$$

In order to avoid the appearance of the square roots, it proved to be more convenient to use the equalities

$$
\frac{\partial \mathcal{F}}{\partial \mathcal{D}_{a b} q}=-i \frac{\bar{\lambda}^{a b}+\frac{1}{2} \bar{\lambda}^{2} \lambda^{a b}}{1-\frac{1}{4} \lambda^{2} \bar{\lambda}^{2}}, \quad \frac{\partial \mathcal{F}}{\partial \mathcal{D}_{a b} \bar{q}}=i \frac{\lambda^{a b}+\frac{1}{2} \lambda^{2} \bar{\lambda}^{a b}}{1-\frac{1}{4} \lambda^{2} \bar{\lambda}^{2}}
$$

Performing a straightforward calculation one gets

$$
\delta[-\operatorname{det} \mathcal{E}(1+\mathcal{F})]=2 i \epsilon^{c} \operatorname{det} \mathcal{E}\left(\mathcal{D}_{a b} \psi_{c} \mathcal{D}^{a b} \bar{q}-2 \mathcal{D}_{a m} \psi^{m} \mathcal{D}_{c}^{a} \bar{q}\right)-2 \epsilon^{c} \operatorname{det} \mathcal{E} \bar{\lambda}_{c m} \mathcal{D}_{a b} \psi^{m} \mathcal{D}^{a d} q \mathcal{D}_{d}^{b} \bar{q}
$$

Similarly, one may find the variation of the integrand of the action $S_{W Z}$ (up to the surface terms disappearing after integration over $\left.d^{3} x\right)$

$$
\delta \mathcal{L}_{W Z}=-2 \beta \epsilon^{c} \operatorname{det} \mathcal{E}\left[\left(\psi^{k} \mathcal{D}_{a b} \bar{\psi}_{k}-\bar{\psi}^{k} \mathcal{D}_{a b} \psi_{k}\right) \mathcal{D}^{a d} \psi_{c} \mathcal{D}_{d}^{b} \bar{q}-\bar{\lambda}_{c m} \mathcal{D}_{a b} \psi^{m} \mathcal{D}^{a d} q \mathcal{D}_{d}^{b} \bar{q}\right]
$$

Now, it is a matter of quite lengthly, but again straightforward calculations, to check that the sum of variations (6.44) and (6.45) is a surface term if

$$
\beta=1
$$

Thus, we conclude that the action of the supermembrane in $D=5$, which is invariant with respect to both unbroken and broken supersymmetries, has the form

$$
\begin{aligned}
S & =2 \int d^{3} x-\int d^{3} x \operatorname{det} \mathcal{E}\left(1+\sqrt{\left(1-\mathcal{D}_{a b} q \mathcal{D}^{a b} \bar{q}\right)^{2}-\left(\mathcal{D}_{a b} q \mathcal{D}^{a b} q\right)\left(\mathcal{D}_{c d} \bar{q} \mathcal{D}^{c d} \bar{q}\right)}\right) \\
& +i \int d^{3} x \operatorname{det} \mathcal{E}\left(\psi^{m} \mathcal{D}_{a b} \bar{\psi}_{m}-\bar{\psi}^{m} \mathcal{D}_{a b} \psi_{m}\right) \mathcal{D}^{a c} q \mathcal{D}_{c}^{b} \bar{q}
\end{aligned}
$$

\subsection{Dualization of the scalars: vector and double vector supermultiplets}

Due to the duality between scalar field, entering the action with the space-time derivatives only, and gauge field strength in $d=3$, the actions for the vector (one scalar dualized) and the double vector (both scalars dualized) 
supermultiplets can be easily obtained within the coset approach. Before performing such dualizations, let us rewrite the action (6.47) in the vector notations. If we introduce the quantity

$$
\mathcal{G}_{a b}=\frac{1}{\sqrt{2}}\left(\psi^{m} \mathcal{D}_{a b} \bar{\psi}_{m}-\bar{\psi}^{m} \mathcal{D}_{a b} \psi_{m}\right)
$$

then only vector indices show up in the action. Passing to the vector notation, we get

$$
\begin{aligned}
S & =2 \int d^{3} x-\int d^{3} x \operatorname{det} \mathcal{E}\left(1+\sqrt{\left(1-\mathcal{D}_{I} q \mathcal{D}_{I} \bar{q}\right)^{2}-\left(\mathcal{D}_{I} q \mathcal{D}_{I} q\right)\left(\mathcal{D}_{J} \bar{q} \mathcal{D}_{J} \bar{q}\right)}\right) \\
& +i \int d^{3} x \operatorname{det} \mathcal{E} \epsilon^{I J K} \mathcal{G}_{I} \mathcal{D}_{J} q \mathcal{D}_{K} \bar{q}
\end{aligned}
$$

where

$$
\mathcal{D}_{I}=\left(\mathcal{E}^{-1}\right)_{I}^{J} \partial_{J}, \quad \mathcal{E}_{I}^{J}=\delta_{I}^{J}-\frac{1}{\sqrt{2}}\left(\sigma^{J}\right)_{a b}\left(\psi^{a} \partial_{I} \bar{\psi}^{b}+\bar{\psi}^{a} \partial_{I} \psi^{b}\right)
$$

\subsubsection{Vector supermultiplet}

The vector $N=2, d=3$ supermultiplet includes one scalar and one gauge fields among the physical bosonic components. Thus, we have to dualize one of the scalar components in the action (6.49). To perform dualization, one has to pass to a pair of real bosons $\{u, v\}$

$$
q=\frac{1}{2}(u+i v), \quad \bar{q}=\frac{1}{2}(u-i v) .
$$

In terms of the newly defined scalars, the action (6.49) reads

$$
\begin{aligned}
S & =2 \int d^{3} x-\int d^{3} x \operatorname{det} \mathcal{E}\left[1+\sqrt{\left(1-\frac{1}{2} \mathcal{D}_{I} u \mathcal{D}_{I} u\right)\left(1-\frac{1}{2} \mathcal{D}_{J} v \mathcal{D}_{J} v\right)-\frac{1}{4}\left(\mathcal{D}_{I} u \mathcal{D}_{I} v\right)^{2}}\right] \\
& +\frac{1}{2} \int d^{3} x \operatorname{det} \mathcal{E} \epsilon^{I J K} \mathcal{G}_{I} \mathcal{D}_{J} u \mathcal{D}_{K} v .
\end{aligned}
$$

The equation of motion for the bosonic field $v$ has the form

$$
\partial_{I}\left(\operatorname{det} \mathcal{E}\left(\mathcal{E}^{-1}\right)_{J}^{I} V_{J}\right)=0, \quad V_{I}=\widetilde{V}_{I}+\frac{1}{2} \epsilon_{I J K} G_{J} \mathcal{D}_{K} u
$$

where

$$
\widetilde{V}_{I}=\frac{\left(1-\frac{1}{2} \mathcal{D} u \cdot \mathcal{D} u\right) \mathcal{D}_{I} v+\frac{1}{2} \mathcal{D} u \cdot \mathcal{D} v \mathcal{D}_{I} u}{2 \sqrt{\left(1-\frac{1}{2} \mathcal{D} u \cdot \mathcal{D} u\right)\left(1-\frac{1}{2} \mathcal{D} v \cdot \mathcal{D} v\right)-\frac{1}{4}(\mathcal{D} u \cdot \mathcal{D} v)^{2}}}
$$

Then, one may find that

$$
\mathcal{D}_{I} v=\frac{2 \widetilde{V}_{I}-\widetilde{V} \cdot \mathcal{D} u \mathcal{D}_{I} u}{\sqrt{1-\frac{1}{2} \mathcal{D} u \cdot \mathcal{D} u+2 \widetilde{V} \cdot \widetilde{V}-(\widetilde{V} \cdot \mathcal{D} u)^{2}}}
$$

Performing the Rauth transformation over the bosonic field $v$, we finally get

$$
\tilde{S}=2 \int d^{3} x-\int d^{3} x \operatorname{det} \mathcal{E}\left(1+\sqrt{1-\frac{1}{2} \mathcal{D} u \cdot \mathcal{D} u+2 \widetilde{V} \cdot \widetilde{V}-(\widetilde{V} \cdot \mathcal{D} u)^{2}}\right) .
$$

This is the action for the $N=2, d=3$ vector supermultiplet which possesses an additional, spontaneously broken $N=2$ supersymmetry.

One should stress that the real field strength is defined in (6.53), but the action has a much simpler structure written in terms of $\widetilde{V}_{I}$. 


\subsubsection{Double vector supermultiplet}

In order to obtain a double vector supermultiplet, one may dualize both scalars in the action (6.49). As the first step, one has to find the equations of motion for the scalar fields

$$
\partial_{I}\left(\operatorname{det} \mathcal{E}\left(\mathcal{E}^{-1}\right)_{J}^{I} V^{J}\right)=0, \quad \partial_{I}\left(\operatorname{det} \mathcal{E}\left(\mathcal{E}^{-1}\right)_{J}^{I} \bar{V}^{J}\right)=0,
$$

where

$$
V_{I}=\widetilde{V}_{I}-i \epsilon_{I J K} G_{J} \mathcal{D}_{k} \bar{q}, \quad \widetilde{V}_{I}=\frac{(1-\mathcal{D} q \cdot \mathcal{D} \bar{q}) \mathcal{D}_{I} \bar{q}+(\mathcal{D} \bar{q} \cdot \mathcal{D} \bar{q}) \mathcal{D}_{I} q}{\sqrt{(1-\mathcal{D} q \cdot \mathcal{D} \bar{q})^{2}-(\mathcal{D} q \cdot \mathcal{D} q)(\mathcal{D} \bar{q} \cdot \mathcal{D} \bar{q})}}
$$

After a standard machinery with the Rauth transformations we finally get the action

$$
\widehat{S}=2 \int d^{3} x-\int d^{3} x \operatorname{det} \mathcal{E}\left[1+\sqrt{(1+\widetilde{V} \cdot \overline{\widetilde{V}})^{2}-\widetilde{V}^{2} \overline{\widetilde{V}}^{2}}-i \epsilon_{I J K} G_{I} \widetilde{V}_{J} \overline{\widetilde{V}}_{K}\right]
$$

The bosonic sector of this action coincides with that constructed in 25]. Again, the simplest form of the action is achieved with the help of $\widetilde{V}_{I}$ variables which are related with field strengths as in (6.57), (6.58).

\section{Conclusion}

In this paper, using a remarkable connection between the partial breaking of global supersymmetry, the coset approach, which realized the specific pattern of supersymmetry breaking, and the Nambu-Goto actions for the extended object, we have reviewed the construction of the on-shell component actions for the superparticle in $D=3$ realizing $N=4 \cdot 2^{k} \rightarrow N=2 \cdot 2^{k}$ pattern of supersymmetry breaking, for the superparticle in $D=5$ with the $N=16$ supersymmetry broken down to $N=8$ one, for the $N=1, D=5$ supermembrane and its dual cousins, and for the $N=1$ supermembrane in $D=4$. Of course, such actions can be obtained by dimensional reduction from the higher dimension actions or even from the known superspace actions. Nevertheless, if we pay more attention to the spontaneously broken supersymmetry and, thus, use the corresponding covariant derivatives, together with the proper choice of the components, the resulting action can be drastically simplified. So, the implications of our results are threefold:

- we demonstrated that the coset approach can be used far beyond the construction of the superfield equations of motion, if we are interested in the component actions,

- we showed that there is a rather specific choice of the superfields and their components which drastically simplifies the component action,

- we argued that the broken supersymmetry fixed the on-shell component action up to some constants, while the role of the unbroken supersymmetry is just to fix these constants.

The application of our approach is not limited to the cases of P-branes only. Different types of D-branes could be also considered in a similar manner. However, once we are dealing with the field strengths, which never show up as the coordinates of the coset space, the proper choice of the components becomes very important. In particular, the Born-Infeld-Nambu-Goto action (6.52), we constructed by the dualization of one scalar field, has a nice, compact form in terms of the "covariant" field strength $\widetilde{V}_{I}$ which is related with the "genuine" field strength, obeying the Bianchi identity, in a rather complicated way (6.53). The same is also true for the Born-Infeld type action (6.59). In order to clarify the nature of these variables, one has to consider the corresponding patterns of the supersymmetry breaking (with one, or without central charges in the $N=4, d=3$ Poincaré superalgebra (A.16) independently. In this respect, the detailed analysis of $N=2 \rightarrow N=1$ supersymmetry breaking in $d=4$ seems to be much more interesting, being a preliminary step to the construction of $N=4$ Born-Infeld action [16, 12, 14, and/or to the action describing partial breaking of $N=1, D=10$ supersymmetry with the hypermultiplet as the Goldstone superfield. 
In this paper we also showed that the on-shell component actions for superparticle have the universal form

$$
S=\alpha \int d t+(1-\alpha) \int d t \mathcal{E}-\int d t \mathcal{E} \sqrt{1-\beta \mathcal{D}_{t} q \mathcal{D}_{t} q} .
$$

With our approach, we explicitly constructed such actions for the superparticles in $D=3$ realizing $N=4 \cdot 2^{k} \rightarrow$ $N=2 \cdot 2^{k}$ pattern of supersymmetry breaking, and in $D=5$ with the $N=16$ supersymmetry broken down to $N=8$ one. It was shown that the corresponding component on-shell actions are invariant under both unbroken and broken supersymmetry. In the considered models only the equality of both unbroken and broken supersymmetries was essential, and their number did not play any role, we expect that all superparticle models with one half partial breaking of global supersymmetry can be constructed similarly, confirming, thereby, its universality.

One possible application of this method is the construction of models with partial breaking of global supersymmetry in cases when $d>2$, where the superspace actions are known (see, e.g., [4, 5, 6, 7]). We assume that these actions derived with our method will have a more simple and understandable form.

It would be quite instructive to understand which new features will appear when we will replace the trivial, flat target space by, for example, the AdS one [26. It seems that the strategy will be the same, and we are planning to report the corresponding results elsewhere.

\section{Acknowledgements}

We thank our co-authors Nikolay Kozyrev and Armen Yeranyan with whom some of the results mentioned here were obtained. We wish to acknowledge useful discussions with them, as well as with S. Kuzenko and D. Sorokin. This work was partially supported by RFBR grants 12-02-00517-a, 13-02-91330-NNIO-a and 13-02-90602 Apm-a, as well as by the ERC Advanced Grant no. 226455 "Supersymmetry, Quantum Gravity and Gauge Fields" (SUPERFIELDS).

\section{Appendix A. Superalgebra, coset space, transformations and Cartan forms}

In this appendix we collected some formulas from the paper 9] where the nonlinear realization of $N=1, D=4$ Poincaré group in its coset over $d=3$ Lorentz group $S O(1,2)$ was constructed.

In $d=3$ notation the $N=1, D=4$ Poincaré superalgebra contains the following set of generators:

$$
\mathrm{N}=2, \mathrm{~d}=3 \mathrm{SUSY} \propto\left\{Q_{a}, P_{a b}, S_{a}, Z, M_{a b}, K_{a b}\right\},
$$

$a, b=1,2$ being the $d=3 S L(2, R)$ spinor indices 7 . Here, $P_{a b}$ and $Z$ are $D=4$ translation generators, $Q_{a}$ and $S_{a}$ are the generators of super-translations, the generators $M_{a b}$ form $d=3$ Lorentz algebra $s o(1,2)$, while the generators $K_{a b}$ belong to the coset $S O(1,3) / S O(1,2)$. The basic anticommutation relations read

$$
\left\{Q_{a}, Q_{b}\right\}=P_{a b}, \quad\left\{Q_{a}, S_{b}\right\}=\epsilon_{a b} Z, \quad\left\{S_{a}, S_{b}\right\}=P_{a b} .
$$

The coset element was defined in [9] as

$$
g=e^{x^{a b} P_{a b}} e^{\theta^{a} Q_{a}} e^{q Z} e^{\psi^{a} S_{a}} e^{\Lambda^{a b} K_{a b}} .
$$

Here, $x^{a b}, \theta^{a}$ are $N=1, d=3$ superspace coordinates, while the remaining coset parameters are Goldstone superfields, $\boldsymbol{q}=\boldsymbol{q}(x, \theta), \boldsymbol{\psi}^{a} \equiv \boldsymbol{\psi}^{a}(x, \theta), \boldsymbol{\Lambda}^{a b}=\boldsymbol{\Lambda}^{a b}(x, \theta)$.

The transformation properties of the coordinates and superfields with respect to all symmetries can be found by acting from the left on the coset element (A.3) by the different elements of $N=1, D=4$ supergroup. They have the following explicit form:

\footnotetext{
${ }^{7}$ The indices are raised and lowered as follows: $V^{a}=\epsilon^{a b} V_{b}, V_{b}=\epsilon_{b c} V^{c}, \epsilon_{a b} \epsilon^{b c}=\delta_{a}^{c}$.
} 
- Translations and Unbroken supersymmetry $\left(g_{0}=\exp \left(a^{a b} P_{a b}+\eta^{a} Q_{a}\right)\right)$

$$
\delta x^{a b}=a^{a b}-\frac{1}{4} \eta^{a} \theta^{b}-\frac{1}{4} \eta^{b} \theta^{a}, \quad \delta \theta^{a}=\eta^{a} .
$$

- Broken supersymmetry $\left(g_{0}=\exp \left(\xi^{a} S_{a}\right)\right)$

$$
\delta x^{a b}=-\frac{1}{4} \xi^{a} \boldsymbol{\psi}^{b}-\frac{1}{4} \xi^{b} \boldsymbol{\psi}^{a}, \quad \delta \boldsymbol{q}=\xi^{a} \theta_{a}, \quad \delta \boldsymbol{\psi}^{a}=\xi^{a} .
$$

- $K$ transformations $\left(g_{0}=\exp \left(r^{a b} K_{a b}\right)\right)$

$$
\begin{aligned}
& \delta x^{a b}=-2 \boldsymbol{q} r^{a b}-\frac{1}{2} \theta_{c} r^{c a} \boldsymbol{\psi}^{b}-\frac{1}{2} \theta_{c} r^{c b} \boldsymbol{\psi}^{a}+\frac{1}{2} \theta^{a} r^{b c} \boldsymbol{\psi}_{c}+\frac{1}{2} \theta^{b} r^{a c} \boldsymbol{\psi}_{c}, \\
& \delta \theta^{a}=-2 r^{a b} \boldsymbol{\psi}_{b}, \quad \delta \boldsymbol{q}=-4 r_{a b} x^{a b}, \quad \delta \boldsymbol{\psi}^{a}=2 r^{a b} \theta_{b}, \quad \delta \boldsymbol{\lambda}^{a b}=r^{a b}-4 \boldsymbol{\lambda}^{a c} r_{c d} \boldsymbol{\lambda}^{d b} .
\end{aligned}
$$

- Broken $Z$-translations $\left(g_{0}=\exp (c Z)\right)$

$$
\delta \boldsymbol{q}=c .
$$

- The $d=3$ Lorentz group $S O(1,2) \sim S L(2, R)$ acts as rotations of the spinor indices.

In (A.6) the coordinates of the stereographic parametrization of the coset $S O(1,3) / S O(1,2)$ have been defined as

$$
\boldsymbol{\lambda}^{a b}=\frac{\tanh \left(\sqrt{2 \Lambda^{2}}\right)}{\sqrt{2 \Lambda^{2}}} \Lambda^{a b}, \quad \tanh ^{2}\left(\sqrt{2 \Lambda^{2}}\right) \equiv 2 \boldsymbol{\lambda}^{2}, \quad \Lambda^{2} \equiv \boldsymbol{\Lambda}_{a b} \Lambda^{a b}, \quad \lambda^{2} \equiv \boldsymbol{\lambda}_{a b} \boldsymbol{\lambda}^{a b} .
$$

The most important objects in the coset are the Cartan forms

$$
g^{-1} d g=\Omega_{Q}+\Omega_{P}+\Omega_{Z}+\Omega_{S}+\Omega_{K}+\Omega_{M} .
$$

In what follows we will need only the forms $\Omega_{Q}, \Omega_{P}, \Omega_{Z}$ and $\Omega_{S}$ which were constructed in $[9]$

$$
\begin{aligned}
\Omega_{Z} & =\frac{1+2 \boldsymbol{\lambda}^{2}}{1-2 \boldsymbol{\lambda}^{2}}\left[d \hat{\boldsymbol{q}}+\frac{4}{1+2 \boldsymbol{\lambda}^{2}} \boldsymbol{\lambda}_{a b} d \hat{x}^{a b}\right] Z \\
\Omega_{P} & \equiv \Omega_{P}^{a b} P_{a b}=\left[d \hat{x}^{a b}+\frac{2}{1-2 \boldsymbol{\lambda}^{2}} \boldsymbol{\lambda}^{a b}\left(d \hat{\boldsymbol{q}}+2 \boldsymbol{\lambda}_{c d} d \hat{x}^{c d}\right)\right] P_{a b}, \\
\Omega_{Q} & \equiv \Omega_{Q}^{a} Q_{a}=\frac{1}{\sqrt{1-2 \boldsymbol{\lambda}^{2}}}\left[d \theta^{a}+2 \boldsymbol{\lambda}^{a b} d \boldsymbol{\psi}_{b}\right] Q_{a}, \\
\Omega_{S} & \equiv \Omega_{S}^{a} S_{a}=\frac{1}{\sqrt{1-2 \boldsymbol{\lambda}^{2}}}\left[d \boldsymbol{\psi}^{a}-2 \boldsymbol{\lambda}^{a b} d \theta_{b}\right] S_{a} . \\
d \hat{x}^{a b} & \equiv d x^{a b}+\frac{1}{4} \theta^{a} d \theta^{b}+\frac{1}{4} \theta^{b} d \theta^{a}+\frac{1}{4} \boldsymbol{\psi}^{a} d \boldsymbol{\psi}^{b}+\frac{1}{4} \boldsymbol{\psi}^{b} d \boldsymbol{\psi}^{a}, \quad d \hat{\boldsymbol{q}} \equiv d \boldsymbol{q}+\boldsymbol{\psi}_{a} d \theta^{a} .
\end{aligned}
$$

Note, that all Cartan forms, except for $\Omega_{M}$, transform homogeneously under all symmetries.

Having at hands the Cartan forms, one may construct the "semi-covariant" (covariant with respect to $d=3$ Lorentz, unbroken and broken supersymmetries only) as

$$
d \hat{x}^{a b} \nabla_{a b}+d \theta^{a} \nabla_{a}=d x^{a b} \frac{\partial}{\partial x^{a b}}+d \theta^{a} \frac{\partial}{\partial \theta^{a}} .
$$

Explicitly, they read [9]

$$
\nabla_{a b}=\left(E^{-1}\right)_{a b}^{c d} \partial_{c d}, \quad \nabla_{a}=D_{a}+\frac{1}{2} \boldsymbol{\psi}^{b} D_{a} \boldsymbol{\psi}^{c} \nabla_{b c}=D_{a}+\frac{1}{2} \boldsymbol{\psi}^{b} \nabla_{a} \boldsymbol{\psi}^{c} \partial_{b c},
$$


where

$$
\begin{aligned}
& D_{a}=\frac{\partial}{\partial \theta^{a}}+\frac{1}{2} \theta^{b} \partial_{a b}, \quad\left\{D_{a}, D_{b}\right\}=\partial_{a b}, \\
& E_{a b}^{c d}=\frac{1}{2}\left(\delta_{a}^{c} \delta_{b}^{d}+\delta_{a}^{d} \delta_{b}^{c}\right)+\frac{1}{4}\left(\boldsymbol{\psi}^{c} \partial_{a b} \boldsymbol{\psi}^{d}+\boldsymbol{\psi}^{d} \partial_{a b} \boldsymbol{\psi}^{c}\right), \\
& \left(E^{-1}\right)_{a b}^{c d}=\frac{1}{2}\left(\delta_{a}^{c} \delta_{b}^{d}+\delta_{a}^{d} \delta_{b}^{c}\right)-\frac{1}{4}\left(\boldsymbol{\psi}^{c} \nabla_{a b} \boldsymbol{\psi}^{d}+\boldsymbol{\psi}^{d} \nabla_{a b} \boldsymbol{\psi}^{c}\right) .
\end{aligned}
$$

These derivatives obey the following algebra:

$$
\begin{aligned}
& {\left[\nabla_{a b}, \nabla_{c d}\right]=-\nabla_{a b} \psi^{m} \nabla_{c d} \boldsymbol{\psi}^{n} \nabla_{m n}, \quad\left[\nabla_{a b}, \nabla_{c}\right]=\nabla_{a b} \boldsymbol{\psi}^{m} \nabla_{c} \boldsymbol{\psi}^{n} \nabla_{m n},} \\
& \left\{\nabla_{a}, \nabla_{b}\right\}=\nabla_{a b}+\nabla_{a} \boldsymbol{\psi}^{m} \nabla_{b} \boldsymbol{\psi}^{n} \nabla_{m n} .
\end{aligned}
$$

\section{Appendix B}

In this Appendix we will prove the invariance of the supermembrane action (5.10) under broken and unbroken supersymmetries. The proof for the broken supersymmetry is the easiest one and we will start with this invariance.

\section{Broken supersymmetry}

Under spontaneously broken $S^{a}$ supersymmetry our coordinates and the physical components transform as in (A.5)

$$
\delta x^{a b}=-\frac{1}{4} \xi^{a} \psi^{b}-\frac{1}{4} \xi^{b} \psi^{a}, \quad \delta q=0, \quad \delta \psi^{a}=\xi^{a} .
$$

One may immediately check that the $\theta=0$ part of the covariant differential $d \hat{x}^{a b}$, defined in (A.10)

$$
d \hat{x}^{a b}=d x^{a b}+\frac{1}{4} \psi^{a} d \psi^{b}+\frac{1}{4} \psi^{b} d \psi^{a}
$$

is invariant under the transformations (B.1). Therefore, the covariant derivatives $\mathcal{D}_{a b}=\left.\nabla_{a b}\right|_{\theta=0}$ (A.12) are also invariant under broken supersymmetry transformations. Now, for the active form of the transformations $\left(\delta^{*} \phi=\phi^{\prime}(x)-\phi(x)\right)$ we have

$$
\begin{aligned}
& \delta_{S}^{*} \mathcal{D}^{a b} q=\frac{1}{2} \xi^{c} \psi^{d} \partial_{c d} \mathcal{D}^{a b} q \quad \Rightarrow \quad \delta_{S}^{*} \mathcal{F}(\mathcal{D} q \cdot \mathcal{D} q)=\frac{1}{2} \xi^{a} \psi^{b} \partial_{a b} \mathcal{F}, \\
& \delta_{S}^{*} \psi^{a}=\xi^{a}+\frac{1}{2} \xi^{c} \psi^{d} \partial_{c d} \psi^{a}, \quad \delta_{S}^{*} \mathcal{D}^{a b} \psi^{c}=\frac{1}{2} \xi^{d} \psi^{e} \partial_{d e} \mathcal{D}^{a b} \psi^{c},
\end{aligned}
$$

and, therefore,

$$
\delta_{S}^{*} \operatorname{det} \mathcal{E}=\frac{1}{2} \xi^{a} \mathcal{D}_{a b} \psi^{b}-\frac{1}{8} \xi^{d} \psi_{d} \mathcal{D}^{a b} \psi^{c} \mathcal{D}_{a b} \psi_{c}+\frac{1}{2} \xi^{c} \psi^{d} \partial_{c d} \operatorname{det} \mathcal{E} .
$$

Thus, the integrand in the action (5.7) transforms as follows:

$$
\begin{aligned}
\delta_{S}^{*}(\operatorname{det} \mathcal{E} \mathcal{F}) & =\left(\frac{1}{2} \xi^{a} \mathcal{D}_{a b} \psi^{b}-\frac{1}{8} \xi^{d} \psi_{d} \mathcal{D}^{a b} \psi^{c} \mathcal{D}_{a b} \psi_{c}\right) \mathcal{F}+\frac{1}{2} \xi^{c} \psi^{d} \partial_{c d}(\operatorname{det} \mathcal{E} \mathcal{F}) \\
& =\left(\frac{1}{2} \xi^{a} \mathcal{D}_{a b} \psi^{b}-\frac{1}{8} \xi^{d} \psi_{d} \mathcal{D}^{a b} \psi^{c} \mathcal{D}_{a b} \psi_{c}-\frac{1}{2} \xi^{c} \partial_{c d} \psi^{d} \operatorname{det} \mathcal{E}\right) \mathcal{F}
\end{aligned}
$$

It is a matter of direct calculations to check that the expression in the parentheses in (B.5) is zero. Thus, the action (5.7), as well as the action (5.10), are indeed invariant under spontaneously broken supersymmetry. 


\section{Unbroken supersymmetry}

It is funny, but in contrast with the superfield approach in which unbroken supersymmetry is manifest, to prove the invariance of the component action (5.10) under unbroken supersymmetry is a rather complicated task.

Under unbroken $Q^{a}$ supersymmetry the covariant derivatives $\nabla_{a b}, \nabla_{a}$ (A.12) are invariant by construction. Therefore, the objects $\nabla_{a b} \psi_{c}, \nabla_{a b} q$ are the superfields with the standard transformation properties

$$
\begin{aligned}
& \delta_{Q}^{*} \psi^{a}=-\left.\eta^{b}\left(D_{b} \boldsymbol{\psi}^{a}\right)\right|_{\theta=0}=2 \eta^{b}\left(\lambda_{b}{ }^{a}-\frac{1}{2} \psi^{m} \lambda_{b}{ }^{n} \partial_{m n} \psi^{a}\right) \\
& \delta_{Q}^{*} \mathcal{D}_{a b} \psi_{c}=-\left.\eta^{d}\left(D_{d} \nabla_{a b} \boldsymbol{\psi}_{c}\right)\right|_{\theta=0}=-\eta^{d}\left(2 \mathcal{D}_{a b} \psi^{m} \lambda_{d}{ }^{n} \mathcal{D}_{m n} \psi_{c}-2 \mathcal{D}_{a b} \lambda_{d c}+\psi^{m} \lambda_{d}{ }^{n} \partial_{m n} \mathcal{D}_{a b} \psi_{c}\right), \\
& \delta_{Q}^{*} \mathcal{D}_{a b} q=-\left.\eta^{c}\left(D_{c} \nabla_{a b} \boldsymbol{q}\right)\right|_{\theta=0}=-\eta^{c}\left(\frac{1-2 \lambda^{2}}{1+2 \lambda^{2}} \mathcal{D}_{a b} \psi_{c}+\psi^{m} \lambda_{c}{ }^{n} \partial_{m n} \mathcal{D}_{a b} q\right)
\end{aligned}
$$

Therefore,

$$
\begin{aligned}
\delta_{Q}^{*} \operatorname{det} \mathcal{E} & =\eta^{c}{\lambda_{c}}^{a} \mathcal{D}_{a b} \psi^{b}-\eta^{c} \mathcal{D}_{a b} \lambda^{b}{ }_{c} \psi^{a}+\eta^{c} \lambda_{c}{ }^{n} \psi^{a} \mathcal{D}_{a b} \psi^{m} \mathcal{D}_{m n} \psi^{b}-\frac{1}{4} \eta^{b} \lambda_{b}{ }^{a} \psi_{a} \mathcal{D}^{m n} \psi^{k} \mathcal{D}_{m n} \psi_{k} \\
& -\frac{1}{8} \psi^{2} \eta^{d} \lambda_{d}{ }^{b} \mathcal{D}_{b c} \psi^{c} \mathcal{D}^{m n} \psi^{k} \mathcal{D}_{m n} \psi_{k}+\frac{1}{4} \psi^{2} \eta^{d} \mathcal{D}_{a b} \lambda_{d c} \mathcal{D}^{a b} \psi^{c}-\eta^{c} \lambda_{c}{ }^{n} \psi^{m} \partial_{m n} \operatorname{det} \mathcal{E}
\end{aligned}
$$

and

$$
\delta_{Q}^{*} \mathcal{F}=-2 \frac{1-2 \lambda^{2}}{1+2 \lambda^{2}} \eta^{c} \mathcal{D}_{a b} \psi_{c} \mathcal{D}^{a b} q \mathcal{F}^{\prime}-\eta^{c} \lambda_{c}{ }^{n} \psi^{m} \partial_{m n} \mathcal{F} .
$$

The $\mathcal{F}^{\prime}$ in $(\mathrm{B} .10)$ denotes the derivative $\mathcal{F}$ over its argument (i.e. over $\mathcal{D} q \cdot \mathcal{D} q$ in our case).

Combining these expressions we will get the following variation of the integrand of our action (5.10):

$$
\delta_{Q}^{*} \mathcal{L}=\delta_{Q}^{*}(\operatorname{det} \mathcal{E} \mathcal{F})=\delta_{Q}^{*} \operatorname{det} \mathcal{E} \mathcal{F}+\operatorname{det} \mathcal{E} \delta_{Q}^{*} \mathcal{F} .
$$

In (B.11) the last terms from $\delta_{Q}^{*} \operatorname{det} \mathcal{E}(\overline{B .9})$ and $\delta_{Q}^{*} \mathcal{F}($ B.10) combine together to produce

$$
-\eta^{a} \lambda_{a}^{b} \psi^{c} \partial_{b c}(\operatorname{det} \mathcal{E F})
$$

Therefore, after integration by parts in this term we will get

$$
\begin{aligned}
\delta_{Q}^{*} \mathcal{L} & =\left(\eta^{c} \lambda_{c}{ }^{a} \mathcal{D}_{a b} \psi^{b}-\eta^{c} \mathcal{D}_{a b} \lambda^{b}{ }_{c} \psi^{a}+\eta^{c} \lambda_{c}{ }^{n} \psi^{a} \mathcal{D}_{a b} \psi^{m} \mathcal{D}_{m n} \psi^{b}-\frac{1}{4} \eta^{b} \lambda_{b}{ }^{a} \psi_{a} \mathcal{D}^{m n} \psi^{k} \mathcal{D}_{m n} \psi_{k}\right. \\
& \left.-\frac{1}{8} \psi^{2} \eta^{d} \lambda_{d}{ }^{b} \mathcal{D}_{b c} \psi^{c} \mathcal{D}^{m n} \psi^{k} \mathcal{D}_{m n} \psi_{k}+\frac{1}{4} \psi^{2} \eta^{d} \mathcal{D}_{a b} \lambda_{d c} \mathcal{D}^{a b} \psi^{c}\right) \mathcal{F} \\
& -2 \frac{1-2 \lambda^{2}}{1+2 \lambda^{2}} \eta^{c} \mathcal{D}_{a b} \psi_{c} \mathcal{D}^{a b} q \mathcal{F}^{\prime} \operatorname{det} \mathcal{E}+\eta^{c} \partial_{m n} \lambda_{c}{ }^{n} \psi^{m} \mathcal{F} \operatorname{det} \mathcal{E}+\eta^{c} \lambda_{c}{ }^{n} \partial_{m n} \psi^{m} \mathcal{F} \operatorname{det} \mathcal{E}
\end{aligned}
$$

Now, one may check that terms with the derivatives of $\lambda_{a b}$ in (B.12) just canceled.

The next step is to substitute into (B.12) the explicit expressions for $\lambda_{a b}(5.2)$ and for $\mathcal{F}$ (5.10)

$$
\lambda_{a b}=\frac{-\frac{1}{2} \mathcal{D}_{a b} q}{1+\sqrt{1-\frac{1}{2} \mathcal{D} q \cdot \mathcal{D} q}}, \quad \mathcal{F}=1+\sqrt{1-\frac{1}{2} \mathcal{D} q \cdot \mathcal{D} q} .
$$

If we note that

$$
\lambda_{a b}=\frac{-\frac{1}{2} \mathcal{D}_{a b} q}{\mathcal{F}} \quad \text { and } \quad \frac{1-2 \lambda^{2}}{1+2 \lambda^{2}}=-\frac{1}{4 \mathcal{F}^{\prime}}
$$


it will be not so strange that after substitution of $(\overline{B .13})$ into $(\overline{B .12})$, the variation $\delta_{Q}^{*} \mathcal{L}$ will not contain any square roots. So, it will read

$$
\begin{aligned}
\delta_{Q}^{*} \mathcal{L} & =-\frac{1}{2} \eta^{c} \mathcal{D}_{c}{ }^{a} q \mathcal{D}_{a b} \psi^{b}-\frac{1}{2} \eta^{c} \mathcal{D}_{c}{ }^{n} q \psi^{a} \mathcal{D}_{a b} \psi^{m} \mathcal{D}_{m n} \psi^{b}+\frac{1}{8} \eta^{b} \mathcal{D}_{b}{ }^{a} q \psi_{a} \mathcal{D}^{c d} \psi^{e} \mathcal{D}_{c d} \psi_{e} \\
& +\frac{1}{16} \psi^{2} \eta^{a} \mathcal{D}_{a}{ }^{b} q \mathcal{D}_{b c} \psi^{c} \mathcal{D}^{d e} \psi^{f} \mathcal{D}_{d e} \psi_{f}+\frac{1}{2} \eta^{c} \mathcal{D}_{a b} \psi_{c} \mathcal{D}^{a b} q \operatorname{det} \mathcal{E} \\
& -\frac{1}{2} \eta^{a} \mathcal{D}_{a}{ }^{b} q \partial_{b c} \psi^{c} \operatorname{det} \mathcal{E} .
\end{aligned}
$$

Substituting now the expression for $\partial_{b c} \psi^{c} \operatorname{det} \mathcal{E}$ from (B.5) and slightly rearranging the terms, we obtain

$$
\begin{aligned}
\delta_{Q} \mathcal{L} & =-\eta^{c} \mathcal{D}_{c}{ }^{a} q \mathcal{D}_{a b} \psi^{b}-\frac{1}{4} \eta^{a} \mathcal{D}_{a}{ }^{b} q \psi_{b} \mathcal{D}^{c d} \psi_{d} \mathcal{D}_{c e} \psi^{e}+\frac{1}{16} \psi^{2} \eta^{a} \mathcal{D}_{a}{ }^{b} q \mathcal{D}_{b c} \psi^{c} \mathcal{D}^{d e} \psi^{f} \mathcal{D}_{d e} \psi_{f} \\
& +\frac{1}{2} \eta^{c} \mathcal{D}_{a b} \psi_{c} \mathcal{D}^{a b} q \operatorname{det} \mathcal{E}
\end{aligned}
$$

Finally, combining the terms in the first line together, we will get the following simple form of the variation of the integrand

$$
\delta_{Q}^{*} \mathcal{L}=-\eta^{c}\left(\mathcal{D}_{c}{ }^{a} q \mathcal{D}_{a b} \psi^{b}-\frac{1}{2} \mathcal{D}^{a b} q \mathcal{D}_{a b} \psi_{c}\right) \operatorname{det} \mathcal{E} .
$$

Unfortunately, further simplifications are not possible. The simplest way to be sure that $\delta_{Q}^{*} \mathcal{L}(\mathrm{B} .17)$ gives zero after integration over $d^{3} x$ is to find the "equation of motion" for $q$ which follows from the "Lagrangian" (B.17)

$$
\frac{\delta}{\delta q} \int d^{3} x \delta_{Q} \mathcal{L}=0
$$

Clearly, the expression (B.18) has to be identically equal to zero if our action is invariant under unbroken supersymmetry. After quite lengthly and tedious, but straightforward calculations, one may show that this is indeed so.

Thus, our action (5.10) is invariant with respect to both broken and unbroken supersymmetries.

\section{Appendix $\mathrm{C}$}

In this Appendix we collected some formulas describing the nonlinear realization of $N=1, D=5$ Poincaré group in its coset over $d=3$ Lorentz group $S O(1,2)$.

In $d=3$ notation the $N=1, d=5$ Poincaré superalgebra contains the following set of generators:

$$
\mathrm{N}=4, \mathrm{~d}=3 \mathrm{SUSY} \propto\left\{P_{a b}, Q_{a}, \bar{Q}_{a}, S_{a}, \bar{S}_{a}, Z, \bar{Z}, M_{a b}, K_{a b}, \bar{K}_{a b}, J\right\},
$$

$a, b=1,2$ being the $d=3 S L(2, R)$ spinor indices 8 . Here, $P_{a b} Z$ and $\bar{Z}$ are $D=5$ translation generators, $Q_{a}, \bar{Q}_{a}$ and $S_{a}, \bar{S}_{a}$ are the generators of super-translations, the generators $M_{a b}$ form $d=3$ Lorentz algebra so(1,2), the generators $K_{a b}$ and $\bar{K}_{a b}$ belong to the coset $S O(1,4) / S O(1,2) \times U(1)$, while $J$ span $u(1)$. The basic (anti)commutation

\footnotetext{
${ }^{8}$ The indices are raised and lowered as follows: $V^{a}=\epsilon^{a b} V_{b}, V_{b}=\epsilon_{b c} V^{c}, \quad \epsilon_{a b} \epsilon^{b c}=\delta_{a}^{c}$.
} 
relations read

$$
\begin{aligned}
& {\left[M_{a b}, M_{c d}\right]=\epsilon_{a d} M_{b c}+\epsilon_{a c} M_{b d}+\epsilon_{b c} M_{a d}+\epsilon_{b d} M_{a c} \equiv(M)_{a b, c d},} \\
& {\left[M_{a b}, P_{c d}\right]=(P)_{a b, c d},\left[M_{a b}, K_{c d}\right]=(K)_{a b, c d},\left[M_{a b}, \bar{K}_{c d}\right]=(\bar{K})_{a b, c d},} \\
& {\left[K_{a b}, \bar{K}_{c d}\right]=\frac{1}{2}(M)_{a b, c d}+2\left(\epsilon_{a c} \epsilon_{b d}+\epsilon_{b c} \epsilon_{a d}\right) J} \\
& {\left[K_{a b}, P_{c d}\right]=-\left(\epsilon_{a c} \epsilon_{b d}+\epsilon_{b c} \epsilon_{a d}\right) Z,\left[\bar{K}_{a b}, P_{c d}\right]=\left(\epsilon_{a c} \epsilon_{b d}+\epsilon_{b c} \epsilon_{a d}\right) \bar{Z},} \\
& {\left[K_{a b}, \bar{Z}\right]=-2 P_{a b},\left[\bar{K}_{a b}, Z\right]=2 P_{a b},} \\
& {\left[M_{a b}, Q_{c}\right]=\epsilon_{a c} Q_{b}+\epsilon_{b c} Q_{a},\left[M_{a b}, \bar{Q}_{c}\right]=(\bar{Q})_{a b, c},\left[M_{a b}, S_{c}\right]=(S)_{a b, c},\left[M_{a b}, \bar{S}_{c}\right]=(\bar{S})_{a b, c},} \\
& {\left[\bar{K}_{a b}, Q_{c}\right]=-(\bar{S})_{a b, c},\left[K_{a b}, \bar{Q}_{c}\right]=(S)_{a b, c},\left[\bar{K}_{a b}, S_{c}\right]=(\bar{Q})_{a b, c},\left[K_{a b}, \bar{S}_{c}\right]=-(Q)_{a b, c},} \\
& {\left[J, Q_{a}\right]=-\frac{1}{2} Q_{a},\left[J, \bar{Q}_{a}\right]=\frac{1}{2} \bar{Q}_{a},\left[J, S_{a}\right]=-\frac{1}{2} S_{a},\left[J, \bar{S}_{a}\right]=\frac{1}{2} \bar{S}_{a},} \\
& {\left[J, K_{a b}\right]=-K_{a b},\left[J, \bar{K}_{a b}\right]=\bar{K}_{a b},[J, Z]=-Z,[J, \bar{Z}]=\bar{Z},} \\
& \left\{Q_{a}, \bar{Q}_{b}\right\}=2 P_{a b},\left\{S_{a}, \bar{S}_{b}\right\}=2 P_{a b},\left\{Q_{a}, S_{b}\right\}=2 \epsilon_{a b} Z,\left\{\bar{Q}_{a}, \bar{S}_{b}\right\}=2 \epsilon_{a b} \bar{Z} .
\end{aligned}
$$

Note, that the generators obey the following conjugation rules:

$$
\begin{aligned}
& \left(P_{a b}\right)^{\dagger}=P_{a b},\left(K_{a b}\right)^{\dagger}=\bar{K}_{a b},\left(M_{a b}\right)^{\dagger}=-M_{a b}, J^{\dagger}=J, Z^{\dagger}=\bar{Z}, \\
& \left(Q_{a}\right)^{\dagger}=\bar{Q}_{a},\left(S_{a}\right)^{\dagger}=\bar{S}_{a} .
\end{aligned}
$$

We define the coset element as follows

$$
g=e^{i x^{a b} P_{a b}} e^{\theta^{a} Q_{a}+\bar{\theta}^{a} \bar{Q}_{a}} e^{i(\boldsymbol{q} Z+\overline{\boldsymbol{q}} \bar{Z})} e^{\boldsymbol{\psi}^{a} S_{a}+\overline{\boldsymbol{\psi}}^{a} \bar{S}_{a}} e^{i\left(\boldsymbol{\Lambda}^{a b} K_{a b}+\overline{\boldsymbol{\Lambda}}^{a b} \bar{K}_{a b}\right)} .
$$

Here, $\left\{x^{a b}, \theta^{a}, \bar{\theta}^{a}\right\}$ are $N=2, d=3$ superspace coordinates, while the remaining coset parameters are Goldstone superfields, $\boldsymbol{q}=\boldsymbol{q}(x, \theta, \bar{\theta}), \overline{\boldsymbol{q}}=\overline{\boldsymbol{q}}(x, \theta, \bar{\theta}), \boldsymbol{\psi}^{a}=\boldsymbol{\psi}^{a}(x, \theta, \bar{\theta}), \overline{\boldsymbol{\psi}}^{a}=\overline{\boldsymbol{\psi}}^{a}(x, \theta, \bar{\theta}), \boldsymbol{\Lambda}^{a b}=\boldsymbol{\Lambda}^{a b}(x, \theta, \bar{\theta}), \overline{\boldsymbol{\Lambda}}^{a b}=\overline{\boldsymbol{\Lambda}}^{a b}(x, \theta, \bar{\theta})$. These $N=2$ superfields obey the following conjugation rules:

$$
\left(x^{a b}\right)^{\dagger}=x^{a b},\left(\theta^{a}\right)^{\dagger}=\bar{\theta}^{a}, \quad \boldsymbol{q}^{\dagger}=\overline{\boldsymbol{q}},\left(\boldsymbol{\psi}^{a}\right)^{\dagger}=\overline{\boldsymbol{\psi}}^{a},\left(\boldsymbol{\Lambda}^{a b}\right)^{\dagger}=\overline{\boldsymbol{\Lambda}}^{a b} .
$$

The transformation properties of the coordinates and superfields with respect to all symmetries can be found by acting from the left on the coset element $g$ (C.4) by the different elements of $N=1, D=5$ Poincaré supergroup. In what follows, we will need only the explicit form only for the broken $(S, \bar{S})$, unbroken $(Q, \bar{Q})$ supersymmetries, and $(K, \bar{K})$ automorphism transformations which read

- Unbroken $(Q)$ supersymmetry $\left(g_{0}=\exp \left(\epsilon^{a} Q_{a}+\bar{\epsilon}^{a} \bar{Q}_{a}\right)\right)$

$$
\delta x^{a b}=i\left(\epsilon^{(a} \bar{\theta}^{b)}+\bar{\epsilon}^{(a} \theta^{a)}\right), \quad \delta \theta^{a}=\epsilon^{a}, \delta \bar{\theta}^{a}=\bar{\epsilon}^{a} .
$$

- Broken $(S)$ supersymmetry $\left(g_{0}=\exp \left(\varepsilon^{a} S_{a}+\bar{\varepsilon}^{a} \bar{S}_{a}\right)\right)$

$$
\delta x^{a b}=i\left(\varepsilon^{(a} \overline{\boldsymbol{\psi}}^{b)}+\bar{\varepsilon}^{(a} \boldsymbol{\psi}^{b)}\right), \quad \delta \boldsymbol{q}=2 i \varepsilon_{a} \theta^{a}, \quad \delta \overline{\boldsymbol{q}}=2 i \bar{\varepsilon}_{a} \bar{\theta}^{a}, \quad \delta \boldsymbol{\psi}^{a}=\varepsilon^{a}, \quad \delta \overline{\boldsymbol{\psi}}^{a}=\bar{\varepsilon}^{a} .
$$

- Automorphism $(K, \bar{K})$ transformations $\left(g_{0}=\exp i\left(a^{a b} K_{a b}+\bar{a}^{a b} \bar{K}_{a b}\right)\right)$

$$
\begin{aligned}
& \delta x^{a b}=-2 i\left(a^{a b} \boldsymbol{q}-\bar{a}^{a b} \overline{\boldsymbol{q}}\right)-2 \theta^{c} \boldsymbol{\psi}_{c} \bar{a}^{a b}+2 \bar{\theta}^{c} \overline{\boldsymbol{\psi}}_{c} a^{a b}, \quad \delta \theta^{a}=-2 i a^{a b} \overline{\boldsymbol{\psi}}_{b}, \quad \delta \bar{\theta}^{a}=2 i \bar{a}^{a b} \boldsymbol{\psi}_{b}, \\
& \delta \boldsymbol{q}=-2 i a^{a b} x_{a b}-2 a^{a b}\left(\theta_{a} \bar{\theta}_{b}-\boldsymbol{\psi}_{a} \overline{\boldsymbol{\psi}}_{b}\right), \quad \delta \boldsymbol{\psi}^{a}=2 i a^{a b} \bar{\theta}_{b}, \\
& \delta \overline{\boldsymbol{q}}=2 i \bar{a}^{a b} x_{a b}-2 \bar{a}^{a b}\left(\theta_{a} \bar{\theta}_{b}-\boldsymbol{\psi}_{a} \overline{\boldsymbol{\psi}}_{b}\right), \quad \delta \overline{\boldsymbol{\psi}}^{a}=-2 i \bar{a}^{a b} \theta_{b} .
\end{aligned}
$$


As the next step of the coset formalism, one can construct the Cartan forms

$$
g^{-1} d g=\Omega_{P}+\Omega_{Q}+\bar{\Omega}_{Q}+\Omega_{Z}+\bar{\Omega}_{Z}+\Omega_{S}+\bar{\Omega}_{S}+\ldots
$$

In what follows we will need only the forms $\left\{\Omega_{P}, \Omega_{Q}, \Omega_{\bar{Q}}, \Omega_{Z}, \Omega_{\bar{Z}}, \Omega_{S}, \Omega_{\bar{S}}\right\}$ which explicitly read

$$
\begin{aligned}
& \Omega_{P}=\left\{(\cosh 2 \sqrt{\boldsymbol{Y}})_{a b}^{c d} \triangle x^{a b}-i\left(\overline{\boldsymbol{\Lambda}}^{a b} \triangle \boldsymbol{q}-\boldsymbol{\Lambda}^{a b} \triangle \overline{\boldsymbol{q}}\right)\left(\frac{\sinh 2 \sqrt{\boldsymbol{Y}}}{\sqrt{\boldsymbol{Y}}}\right)_{a b}^{c d}\right\} P_{c d}, \\
& \Omega_{Q}=\left\{d \theta^{b}(\cos 2 \sqrt{\overline{\boldsymbol{T}}})_{b}^{c}-i d \overline{\boldsymbol{\psi}}^{b} \boldsymbol{\Lambda}_{b}{ }^{a}\left(\frac{\sin 2 \sqrt{\overline{\boldsymbol{T}}}}{\sqrt{\overline{\boldsymbol{T}}}}\right)_{a}^{c}\right\} Q_{c} \\
& \Omega_{Z}=\left\{\triangle \boldsymbol{q}+\left(\overline{\boldsymbol{\Lambda}}^{a b} \triangle \boldsymbol{q}-\boldsymbol{\Lambda}^{a b} \triangle \overline{\boldsymbol{q}}\right)\left(\frac{\cosh 2 \sqrt{\boldsymbol{Y}}-1}{\boldsymbol{Y}}\right)_{a b}^{c d} \boldsymbol{\Lambda}_{c d}+i d x^{a b}\left(\frac{\sinh 2 \sqrt{\boldsymbol{Y}}}{\sqrt{\boldsymbol{Y}}}\right)_{a b}^{c d} \boldsymbol{\Lambda}_{c d}\right\} Z, \\
& \Omega_{S}=\left\{d \boldsymbol{\psi}^{b}(\cos 2 \sqrt{\overline{\boldsymbol{T}}})_{b}^{c}+i d \bar{\theta}^{b} \boldsymbol{\Lambda}_{b}^{a}\left(\frac{\sin 2 \sqrt{\overline{\boldsymbol{T}}}}{\sqrt{\overline{\boldsymbol{T}}})_{a}^{c}}\right\}_{c} S_{c}\right. \\
& \triangle x^{a b}=d x^{a b}-i\left(\theta^{(a} d \bar{\theta}^{b)}+\bar{\theta}^{(a} d \theta^{b)}+\boldsymbol{\psi}^{(a} d \overline{\boldsymbol{\psi}}^{b}+\overline{\boldsymbol{\psi}}^{(a} d \boldsymbol{\psi}^{b}\right) \\
& \triangle \boldsymbol{q}=d \boldsymbol{q}-2 i \boldsymbol{\psi}_{a} d \theta^{a}, \quad \triangle \overline{\boldsymbol{q}}=d \overline{\boldsymbol{q}}-2 i \overline{\boldsymbol{\psi}}_{a} d \bar{\theta}^{a} .
\end{aligned}
$$

Here, we defined matrix-valued functions $\boldsymbol{Y}_{a b}{ }^{c d}, \boldsymbol{T}_{a}{ }^{b}$ and $\overline{\boldsymbol{T}}_{a}{ }^{b}$ as

$$
\boldsymbol{Y}_{a b}{ }^{c d}=\boldsymbol{\Lambda}_{a b} \overline{\boldsymbol{\Lambda}}^{c d}+\overline{\boldsymbol{\Lambda}}_{a b} \boldsymbol{\Lambda}^{c d}, \quad \boldsymbol{T}_{a}{ }^{b}=\boldsymbol{\Lambda}_{a}{ }^{c} \overline{\boldsymbol{\Lambda}}_{c}^{b}, \overline{\boldsymbol{T}}_{a}^{b}=\overline{\boldsymbol{\Lambda}}_{a}^{c} \boldsymbol{\Lambda}_{c}^{b} .
$$

Note, that all these Cartan forms transform homogeneously under all symmetries.

Having at hands the Cartan forms, one may construct the "semi-covariant" (covariant with respect to $d=3$ Lorentz, unbroken and broken supersymmetries only) as

$$
\triangle x^{a b} \nabla_{a b}+d \theta^{a} \nabla_{a}+d \bar{\theta}^{a} \bar{\nabla}_{a}=d x^{a b} \frac{\partial}{\partial x^{a b}}+d \theta^{a} \frac{\partial}{\partial \theta^{a}}+d \bar{\theta}^{a} \frac{\partial}{\partial \bar{\theta}^{a}} .
$$

Explicitly, they read

$$
\begin{aligned}
& \nabla_{a b}=\left(E^{-1}\right)_{a b}^{c d} \partial_{c d} \\
& \nabla_{a}=D_{a}-i\left(\boldsymbol{\psi}^{b} D_{a} \overline{\boldsymbol{\psi}}^{c}+\overline{\boldsymbol{\psi}}^{b} D_{a} \boldsymbol{\psi}^{c}\right) \nabla_{b c}=D_{a}-i\left(\boldsymbol{\psi}^{b} \nabla_{a} \overline{\boldsymbol{\psi}}^{c}+\overline{\boldsymbol{\psi}}^{b} \nabla_{a} \boldsymbol{\psi}^{c}\right) \partial_{b c}
\end{aligned}
$$

where

$$
\begin{aligned}
& D_{a}=\frac{\partial}{\partial \theta^{a}}-i \bar{\theta}^{b} \partial_{a b}, \bar{D}_{a}=\frac{\partial}{\partial \bar{\theta}^{a}}-i \theta^{b} \partial_{a b}, \quad\left\{D_{a}, \bar{D}_{b}\right\}=-2 i \partial_{a b} \\
& E_{a b}{ }^{c d}=\delta_{a}^{(c} \delta_{b}^{d)}-i\left(\boldsymbol{\psi}^{(c} \partial_{a b} \overline{\boldsymbol{\psi}}^{d)}+\overline{\boldsymbol{\psi}}^{(c} \partial_{a b} \boldsymbol{\psi}^{d)}\right) \\
& \left(E^{-1}\right)_{a b}{ }^{c d}=\delta_{a}^{(c} \delta_{b}^{d)}+i\left(\boldsymbol{\psi}^{(c} \nabla_{a b} \overline{\boldsymbol{\psi}}^{d)}+\overline{\boldsymbol{\psi}}^{(c} \nabla_{a b} \boldsymbol{\psi}^{d)}\right) .
\end{aligned}
$$

The derivatives obey the following algebra:

$$
\begin{aligned}
& \left\{\nabla_{a}, \nabla_{b}\right\}=-2 i\left(\nabla_{a} \boldsymbol{\psi}^{c} \nabla_{b} \overline{\boldsymbol{\psi}}^{d}+\nabla_{a} \overline{\boldsymbol{\psi}}^{c} \nabla_{b} \boldsymbol{\psi}^{d}\right) \nabla_{c d} \\
& \left\{\nabla_{a}, \bar{\nabla}_{b}\right\}=-2 i \nabla_{a b}-2 i\left(\nabla_{a} \boldsymbol{\psi}^{c} \bar{\nabla}_{b} \overline{\boldsymbol{\psi}}^{d}+\nabla_{a} \overline{\boldsymbol{\psi}}^{c} \bar{\nabla}_{b} \boldsymbol{\psi}^{d}\right) \nabla_{c d} \\
& {\left[\nabla_{a b}, \nabla_{c}\right]=-2 i\left(\nabla_{a b} \boldsymbol{\psi}^{d} \nabla_{c} \overline{\boldsymbol{\psi}}^{f}+\nabla_{a b} \overline{\boldsymbol{\psi}}^{d} \nabla_{c} \boldsymbol{\psi}^{f}\right) \nabla_{d f}} \\
& {\left[\nabla_{a b}, \nabla_{c d}\right]=2 i\left(\nabla_{a b} \boldsymbol{\psi}^{m} \nabla_{c d} \overline{\boldsymbol{\psi}}^{n}-\nabla_{c d} \boldsymbol{\psi}^{m} \nabla_{a b} \overline{\boldsymbol{\psi}}^{n}\right) \nabla_{m n}}
\end{aligned}
$$


The $d=3$ volume form is defined as

$$
d^{3} x \equiv \epsilon_{I J K} d x^{I} \wedge d x^{J} \wedge d x^{K} \quad \Rightarrow \quad d x^{I} \wedge d x^{J} \wedge d x^{K}=\frac{1}{6} \epsilon^{I J K} d^{3} x .
$$

Transition from the spinor notations to the vector one is set as follows

$$
V^{I} \equiv \frac{i}{\sqrt{2}}\left(\sigma^{I}\right)_{a}{ }^{b} V_{b}{ }^{a} \quad \Rightarrow \quad V_{a}{ }^{b}=-\frac{i}{\sqrt{2}} V^{I}\left(\sigma^{I}\right)_{a}{ }^{b}, \quad V^{a b} V_{a b}=V^{I} V^{I} .
$$

Here we are using the standard set of $\sigma^{I}$ matrices

$$
\sigma^{I} \sigma^{J}=i \epsilon^{I J K} \sigma^{K}+\delta^{I J} \mathcal{I}, \quad\left(\sigma^{I}\right)_{a}{ }^{b}\left(\sigma^{I}\right)_{c}{ }^{d}=2 \delta_{a}{ }^{d} \delta_{c}{ }^{b}-\delta_{a}{ }^{b} \delta_{c}{ }^{d},
$$

were $\epsilon^{I J K}$ obeys relations

$$
\epsilon^{I J K} \epsilon_{I M N}=\delta_{M}^{J} \delta_{N}^{K}-\delta_{N}^{J} \delta_{M}^{K}, \quad \epsilon^{I J K} \epsilon_{I J N}=2 \delta_{N}^{K}, \quad \epsilon^{I J K} \epsilon_{I J K}=6 .
$$

\section{References}

[1] S.R. Coleman, J. Wess, B. Zumino,

Structure of phenomenological Lagrangians. 1,

Phys.Rev. 177 (1969) 2239;

C.G. Callan, Jr., S.R. Coleman, J. Wess, B. Zumino,

Structure of phenomenological Lagrangians. 2,

Phys.Rev. 177 (1969) 2247.

[2] D.V. Volkov,

Phenomenological Lagrangians,

Sov.J.Part.Nucl. 4(1973) 3;

V.I. Ogievetsky,

Nonlinear realizations of internal and space-time symmetries,

In Proceedings of the Xth Winter School of Theoretical Physics in Karpacz, Vol.1, p.117, 1974.

[3] D.V. Volkov and V.P. Akulov,

Possible universal neutrino interaction,

JETP Lett. 16(1972) 438;

Is the neutrino a Goldstone particle?

Phys. Lett. B46(1973) 109.

[4] J. Bagger, A. Galperin,

Matter couplings in partially broken extended supersymmetry,

Phys.Lett. B336 (1994) 25-31; arXiv:hep-th/9406217.

[5] J. Bagger, A. Galperin,

New Goldstone multiplet for partially broken supersymmetry,

Phys.Rev. D55 (1997) 1091; arXiv:hep-th/9608177.

[6] J. Bagger, A. Galperin,

The tensor Goldstone multiplet for partially broken supersymmetry,

Phys.Lett. B412 (1997) 296; arXiv:hep-th/9707061.

[7] M. Rocek, A.A. Tseytlin,

Partial breaking of global D=4 supersymmetry, constrained superfields, and 3-brane actions, Phys.Rev. D59 (1999) 106001; arXiv:hep-th/9811232. 
[8] F. Gonzalez-Rey, I.Y. Park, M. Rocek,

On dual 3-brane actions with partially broken $N=2$ supersymmetry,

Nucl.Phys. B544 (1999) 243; arXiv:hep-th/9811130.

[9] E. Ivanov, S. Krivonos,

$N=1, D=4$ supermembrane in the coset approach,

Phys.Lett B453 (1999) 237, arXiv:hep-th/9901003.

[10] S. Bellucci, E. Ivanov, S. Krivonos,

Superbranes and Super Born-Infeld Theories from Nonlinear Realizations,

Nucl.Phys.Proc.Suppl. 102 (2001) 26; arXiv:hep-th/0103136

[11] S. Bellucci, E. Ivanov, S. Krivonos,

Partial breaking of $N=1, D=10$ supersymmetry,

Phys.Lett. B460 (1999) 348; arXiv: hep-th/9811244,

Partial breaking $N=4$ to $N=2$ : hypermultiplet as a Goldstone superfield,

Fortsch.Phys. 48 (2000)19; arXiv:hep-th/9809190.

[12] S. Bellucci, E. Ivanov, S. Krivonos,

$N=2$ and $N=4$ supersymmetric Born-Infeld theories from nonlinear realizations,

Phys.Lett. B502 (2001) 279; arXiv:hep-th/0012236.

[13] E.A. Ivanov, V.I. Ogievetsky,

The Inverse Higgs Phenomenon in Nonlinear Realizations,

Teor. Mat. Fiz. 25 (1975) 164.

[14] S. Bellucci, E. Ivanov, S. Krivonos,

Towards the complete $N=2$ superfield Born-Infeld action with partially broken $N=4$ supersymmetry,

Phys.Rev. D64 (2001) 025014; arXiv:hep-th/0101195.

[15] M. Aganagic, C. Popescu, J. H. Schwarz, Gauge-Invariant and Gauge-Fixed D-Brane Actions,

Nucl.Phys. B495 (1997) 99; arXiv:hep-th/9612080

[16] E. Bergshoeff, F. Coomans, R. Kallosh, C.S. Shahbazi, A. Van Proeyen, Dirac-Born-Infeld-Volkov-Akulov and Deformation of Supersymmetry,

JHEP 1308 (2013) 100; arXiv:1303.5662.

[17] S. Bellucci, S. Krivonos, A. Sutulin,

Supersymmetric component actions via coset approach,

Phys.Lett. B726 (2013) 497; arXiv:1306.1115.

[18] S. Bellucci, N. Kozyrev, S. Krivonos, A. Sutulin,

Partial breaking of global supersymmetry and super particle actions,

arXiv:1309.3902[hep-th].

[19] S. Bellucci, N. Kozyrev, S. Krivonos, A. Yeranyan,

Supermembrane in $D=5$ : component action,

arXiv:1312.0231[hep-th].

[20] T.E. Clark, M. Nitta,T. ter Veldhuis,

Brane dynamics from nonlinear realizations,

Phys.Rev. D67 (2003) 085026.

[21] M. Hanneaux, L. Mezincescu, A $\sigma$-model interpretation of Green-Schwarz covariant superstring action, Phys.Lett. B152 (1985) 340. 
[22] D. Sorokin,

Superbranes and Superembeddings,

Phys.Rept. 329 (2000) 1; arXiv:hep-th/9906142.

[23] S. Bellucci, E. Ivanov, S. Krivonos, O. Lechtenfeld, $A B C$ of $N=8, d=1$ supermultiplets, Nucl.Phys. B699 (2004) 226; arXiv:hep-th/0406015.

[24] A. Achúcarro, J. Gauntlett, K. Itoh and P.K. Townsend, World-Volume Supersymmetry from Spacetime Supersymmetry of the Four-Dimensional Supermembrane, Nuclear Physics B314 (1989) 129.

[25] E. Ivanov, S. Krivonos, O. Lechtenfeld, Double vector multiplet and partially broken $N=4, D=3$ supersymmetry, Phys.Lett. B487 (2000) 192, arXiv:hep-th/0006017.

[26] S. Bellucci, E. Ivanov, S. Krivonos, Goldstone superfield actions in AdS5 backgrounds, Nucl.Phys. B672 (2003) 123; arXiv:hep-th/0212295. 